MATHEMATICS OF COMPUTATION

Volume 74 , Number 249 , Pages 53-84

S 0025-5718(04)01652-7

Article electronically published on March 23, 2004

\title{
A DISCONTINUOUS GALERKIN METHOD WITH NONOVERLAPPING DOMAIN DECOMPOSITION FOR THE STOKES AND NAVIER-STOKES PROBLEMS
}

\author{
VIVETTE GIRAULT, BÉATRICE RIVIÈRE, AND MARY F. WHEELER
}

\begin{abstract}
A family of discontinuous Galerkin finite element methods is formulated and analyzed for Stokes and Navier-Stokes problems. An inf-sup condition is established as well as optimal energy estimates for the velocity and $L^{2}$ estimates for the pressure. In addition, it is shown that the method can treat a finite number of nonoverlapping domains with nonmatching grids at interfaces.
\end{abstract}

\section{INTRODUCTION}

This paper is devoted to the numerical analysis of a discontinuous Galerkin (DG) method with nonoverlapping domain decomposition, of order $k=1,2$ or 3 for solving the steady incompressible Stokes and Navier-Stokes systems of equations. The finite elements are defined on conforming triangular meshes in each subdomain. In each triangle, the finite elements discretizing the velocity are polynomials of degree $k$ with no continuity requirement between triangles and the finite elements discretizing the pressure are polynomials of degree $k-1$, also totally discontinuous. Boundary conditions are not imposed on the finite element spaces. The viscous part of the operator is put into a variational form with a jump term on all triangle interfaces, so that it is always elliptic. The jump term corrects the discontinuity of the velocity at the interfaces and it corrects the nonzero value of the velocity on the boundary. This paper considers both nonsymmetric and symmetric formulations. In the case where the bilinear form is nonsymmetric, there is no restriction on the coefficient of the jump term.

The zero divergence constraint is imposed by a form that is locally conservative away from subdomain interfaces. This form coincides with the standard form up to an extra jump on interfaces that is introduced for satisfying the inf-sup condition. This extra jump is not needed if there is no domain decomposition. We analyze the discrete Stokes problem by establishing a uniform discrete inf-sup condition for the pressure. This is vital for proving optimal estimates for the velocity and pressure. The nonlinear convection term of the Navier-Stokes equation is discretized by adapting to totally discontinuous velocities the upwind scheme introduced by

Received by the editor March 26, 2002 and, in revised form, May 9, 2003.

2000 Mathematics Subject Classification. Primary 35Q30; Secondary 76D05, 76D07.

Key words and phrases. Discontinuous finite element methods, Navier-Stokes, domain decomposition, nonconforming grids, local mass conservation.

Each author was supported in part by DOD Pet2 Grant and NSF Grants KDI\#DMS-9873326 and ITR\#EIA-0121523. 
Lesaint and Raviart [17] for solving the transport of neutrons. We analyze the nonlinearity by proving uniform $L^{p}$ estimates for the discrete velocity. This enables us to prove the existence of discrete solutions and error estimates.

We assume a fixed number of subdomains. At interfaces of subdomains, the triangulations need not match, but we assume that one is a refinement of the other. This restriction, which does not arise when solving a diffusion or convectiondiffusion equation, is used for proving the inf-sup condition. Relaxing altogether this restriction in the proof does not seem straightforward. But this is not surprising, if we had enforced continuity at subdomain interfaces by a Lagrange multiplier, we would have had two multipliers (the other one being the pressure), and when there is more than one multiplier, these are usually related; see, e.g., Girault, Glowinski, López and Vila [11. However, this is only a sufficient condition and it may not be necessary.

There exist in the literature many finite element approximations of the steady incompressible Stokes and Navier-Stokes problems. The reader can refer to Girault and Raviart [12 and Pironneau 21] for a good description and study of a wide collection of schemes. But to our knowledge, there is very little literature on completely discontinuous Galerkin methods for solving numerically the incompressible Navier-Stokes flows in the primitive variables. Of course, there are the nonconforming finite element schemes of Crouzeix and Raviart [7, Fortin and Soulié [9] and Crouzeix and Falk [6], but their velocities must be continuous at the $k$ Gauss points on triangle sides, whereas we impose here no continuity requirement at all. Thus implementing our scheme is much easier. Moreover, it lends itself readily to nonoverlapping domain decomposition. It has the great advantage over mortar elements in that it does not require Lagrange multipliers on the subdomain boundaries.

We refer to [27, 22, [23, 15, 24] for solving diffusion and convection-diffusion equations by discontinuous Galerkin methods. In [3], Becker, Hansbo and Stenberg discretize a Laplace equation with domain decomposition by means of a symmetric bilinear form and two penalty terms on each subdomain interface. Finally, upwinding the convective term of the Navier-Stokes equations by the Lesaint-Raviart method is now well known. We refer to [12, 21] for a thorough study. The reader can refer to 2] (respectively, [16]) for solving the Stokes (respectively, the NavierStokes) systems by approximating the velocity by discontinuous polynomials that are pointwise divergence-free, approximating the pressure by continuous polynomials. In [5], the authors propose and analyze a discontinuous Galerkin method for the Stokes problem written in terms of velocity, gradient of velocity tensor and pressure.

The outline of the paper is as follows. The Stokes and Navier-Stokes problems are stated and their regularity is discussed in Section 2. The scheme for the Stokes problem is introduced in Section 3. Section 4 is devoted to the proof of the inf-sup condition and Section 5 to a priori estimates for the Stokes problem. Section 6 contains the main points of the discrete Navier-Stokes problem. Conclusions are given in the final section.

\section{Model Stokes and NAVIER-Stokes Problems}

Let $\Omega$ be a Lipschitz domain of $\mathbb{R}^{2}$. Let $\boldsymbol{f} \in H^{-1}(\Omega)^{2}$ and $\mu>0$. The solution $(\boldsymbol{u}, p) \in H^{1}(\Omega)^{2} \times L^{2}(\Omega)$ of the stationary homogeneous Stokes problem for an 
incompressible viscous fluid confined in $\Omega$ satisfies

$$
\begin{aligned}
-\mu \Delta \boldsymbol{u}+\nabla p & =\boldsymbol{f}, \quad \text { in } \Omega \\
\nabla \cdot \boldsymbol{u} & =0, \quad \text { in } \Omega \\
\boldsymbol{u} & =\mathbf{0}, \quad \text { on } \partial \Omega .
\end{aligned}
$$

Since $p$ is uniquely defined up to an additive constant, we also assume that $\int_{\Omega} p=0$. With the above assumptions, this problem has a unique solution $\boldsymbol{u} \in H_{0}^{1}(\Omega)^{2}, p \in$ $L_{0}^{2}(\Omega)[12$, where

$$
\begin{aligned}
& H_{0}^{1}(\Omega)=\left\{v \in H^{1}(\Omega): v=0 \text { on } \partial \Omega\right\} \\
& L_{0}^{2}(\Omega)=\left\{q \in L^{2}(\Omega): \quad \int_{\Omega} q=0\right\} .
\end{aligned}
$$

However, in what follows, we shall need that both the gradient of $\boldsymbol{u}$ and the pressure $p$ have a trace on line segments. For this, it suffices for instance that the data $\boldsymbol{f}$ belong to $L^{4 / 3}(\Omega)^{2}$. Indeed, Grisvard establishes in $[14$ that if $\Omega$ is a Lipschitz polygon (i.e., a polygon with no slits) and $f \in L^{4 / 3}(\Omega)^{2}$, then the solution $(\boldsymbol{u}, p)$ of (2.1) 2.3 belongs to $W^{2,4 / 3}(\Omega)^{2} \times W^{1,4 / 3}(\Omega)$ with continuous dependence on $\|\boldsymbol{f}\|_{L^{4 / 3}(\Omega)}$. Thus each component of the gradient of $\boldsymbol{u}$ has a trace on a line segment $e$, this trace belongs to $W^{1 / 4,4 / 3}(e)$ and by Sobolev's imbedding, $W^{1 / 4,4 / 3}(e) \hookrightarrow$ $L^{2}(e)$. Therefore, the trace of each component of the gradient of $\boldsymbol{u}$ on a line segment $e$ is well defined and belongs to $L^{2}(e)$. The same result holds for the trace of the pressure. Note that both results are sharp.

The Stokes system is a linearized version of the Navier-Stokes system of equations, where (2.1) is replaced by

$$
-\mu \Delta \boldsymbol{u}+\boldsymbol{u} \cdot \nabla \boldsymbol{u}+\nabla p=\boldsymbol{f}, \quad \text { in } \quad \Omega,
$$

and (2.2), (2.3) are unchanged. Here,

$$
\boldsymbol{u} \cdot \nabla \boldsymbol{u}=\sum_{i=1}^{2} u_{i} \frac{\partial \boldsymbol{u}}{\partial x_{i}}
$$

is the convection term. It can be shown that $(2.4),(2.2),(2.3)$ always has a solution (not necessarily unique) $(\boldsymbol{u}, p) \in H_{0}^{1}(\Omega)^{2} \times L_{0}^{2}(\Omega)$; see for example [12, Lions [19] or Temam 25]. As far as regularity is concerned, note that by virtue of the Sobolev imbedding in two dimensions: $H^{1}(\Omega) \hookrightarrow L^{p}(\Omega)$ for any real number $p<\infty$ :

$$
\forall \boldsymbol{v} \in H_{0}^{1}(\Omega), \quad\|\boldsymbol{v}\|_{L^{p}(\Omega)} \leq S_{p}\|\nabla \boldsymbol{v}\|_{L^{2}(\Omega)},
$$

we have that $\boldsymbol{u} \cdot \nabla \boldsymbol{u}$ belongs in particular to $L^{4 / 3}(\Omega)^{2}$. Therefore applying the above regularity of the Stokes problem to

$$
-\mu \Delta \boldsymbol{u}+\nabla p=\boldsymbol{f}-\boldsymbol{u} \cdot \nabla \boldsymbol{u}
$$

we see that, if $\boldsymbol{f}$ belongs to $L^{4 / 3}(\Omega)^{2}$, then every solution $(\boldsymbol{u}, p)$ of (2.4), (2.2), (2.3) belongs also to $W^{2,4 / 3}(\Omega)^{2} \times W^{1,4 / 3}(\Omega)$. Both problems will be analyzed for $\boldsymbol{f} \in L^{4 / 3}(\Omega)^{2}$. But of course optimal error estimates will require more regularity. 


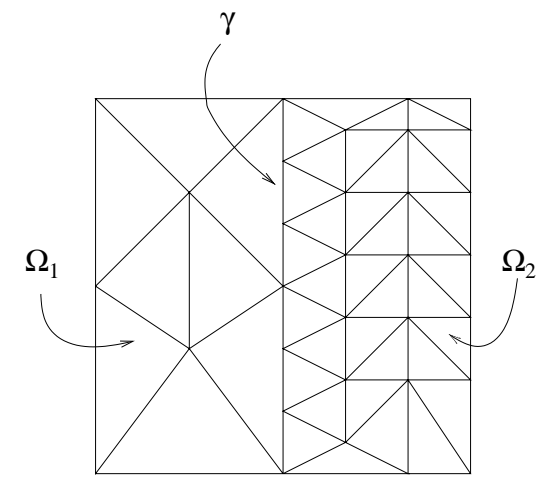

FIgURE 1. Example of two subdomains where $\gamma_{h}^{2}$ is a subgrid of $\gamma_{h}^{1}$.

\section{NOTATION AND PRELIMINARIES}

To simplify, we shall present here a domain decomposition into two subdomains but the analysis below applies to an arbitrary (but fixed) number of subdomains. From now on, we assume that $\Omega$ is a Lipschitz polygon partitioned into two subdomains $\Omega_{1}$ and $\Omega_{2}$, both Lipschitz polygons, with interface $\gamma$ (which is also a polygonal line), i.e., $\Omega=\Omega_{1} \cup \gamma \cup \Omega_{2}$ (see Figure丩). For $i=1,2$, let $\mathcal{E}_{h}^{i}$ be a regular family of triangulations of $\bar{\Omega}_{i}$, consisting of triangles of maximum diameter $h$. Let $h_{E}$ denote the diameter of a triangle $E$ and $\rho_{E}$ the diameter of its inscribed circle. By regular, we mean (see Ciarlet [4]) that there exists a parameter $\sigma>0$ such that

$$
\forall E \in \mathcal{E}_{h}^{i}, \quad i=1,2, \quad \frac{h_{E}}{\rho_{E}}=\sigma_{E} \leq \sigma .
$$

Strictly speaking, we should distinguish all parameters by an index $i$, but we shall usually suppress this index, to alleviate notation. We denote by $\Gamma_{h}^{i}$ the set of all edges of $\mathcal{E}_{h}^{i}$ that do not lie on $\gamma$, set $\Gamma_{h}=\Gamma_{h}^{1} \cup \Gamma_{h}^{2}$ and denote by $\gamma_{h}^{i}$ the set of edges of $\mathcal{E}_{h}^{i}$ that lie on $\gamma$. At the interface $\gamma$, the two meshes $\mathcal{E}_{h}^{i}$ are related by two assumptions specified in Section 4

(i) the assumption $\mathrm{H} 1$ that states that either $\gamma_{h}^{1}$ is a subgrid of $\gamma_{h}^{2}$ or $\gamma_{h}^{2}$ is a subgrid of $\gamma_{h}^{1}$.

(ii) the assumption $\mathrm{H} 2$ of local quasi-uniformity on $\gamma$.

For any nonnegative integer $k$ and number $r \geq 1$, recall the classical Sobolev space on a domain $\mathcal{O} \subset \mathbb{R}^{2}$

$$
W^{k, r}(\mathcal{O})=\left\{v \in L^{r}(\mathcal{O}): \quad \forall|m| \leq k, \quad \partial^{m} v \in L^{r}(\mathcal{O})\right\},
$$

where $\partial^{m} v$ are the partial derivatives of $v$ of order $m$. It is a Banach space for the graph norm, which we denote by $\|\cdot\|_{W^{k, r}(\mathcal{O})}$. In view of the regularity considerations of the previous section, we define

$$
\begin{aligned}
& X=\left\{v \in L^{2}(\Omega): \quad \forall E \in \mathcal{E}_{h},\left.\quad v\right|_{E} \in W^{2,4 / 3}(E)\right\} \\
& M=\left\{q \in L_{0}^{2}(\Omega): \quad \forall E \in \mathcal{E}_{h},\left.\quad q\right|_{E} \in W^{1,4 / 3}(E)\right\} .
\end{aligned}
$$

For an integer $k \geq 0$, the usual Sobolev norm (resp. seminorm) of $H^{k}(\mathcal{O})=W^{k, 2}(\mathcal{O})$ is denoted by $\|\cdot\|_{k, \mathcal{O}}\left(\right.$ resp. $\left.|\cdot|_{k, \mathcal{O}}\right)$, the $L^{2}$ norm corresponding to $k=0$. If $\mathcal{O}=\Omega$ and if there is no ambiguity, we simply write $\|\cdot\|_{k}$ (resp. $|\cdot|_{k}$ ). We refer to Adams 
[1] or Lions and Magenes [18] for the definition of the norm of $H^{s}(\Omega)$, for real $s>0$. The norm associated with $X$ is the "broken" norm

$$
\|\cdot\|_{k}^{2}=\sum_{E \in \mathcal{E}_{h}}\|\cdot\|_{k, E}^{2} \cdot
$$

We denote the product space by $\boldsymbol{X}=X^{2}$. Let $\mathcal{D}(\mathcal{O})$ denote the space of infinitely differentiable functions with compact support on $\mathcal{O}$ and $\mathcal{D}^{\prime}(\mathcal{O})$ the space of distributions on $\mathcal{O}$. We recall that, by definition, for $\boldsymbol{v}=\left(v_{i}\right)_{i}, \nabla \boldsymbol{v}=\left(\frac{\partial v_{i}}{\partial x_{j}}\right)_{i, j}$ and $\nabla \boldsymbol{v} \boldsymbol{n}$ is the product of the matrix $\nabla \boldsymbol{v}$ by the vector $\boldsymbol{n}$. Let $e$ denote a segment of $\Gamma_{h}^{i}$ shared by two triangles $E^{k}$ and $E^{l}$ of $\mathcal{E}_{h}^{i}$; we associate with $e$, once and for all, a unit normal vector $\boldsymbol{n}_{e}$ directed from $E^{k}$ to $E^{l}$ and we define formally the jump and average of a function $\phi$ on $e$ by

$$
[\phi]=\left.\left(\left.\phi\right|_{E^{k}}\right)\right|_{e}-\left.\left(\left.\phi\right|_{E^{l}}\right)\right|_{e}, \quad\{\phi\}=\left.\frac{1}{2}\left(\left.\phi\right|_{E^{k}}\right)\right|_{e}+\left.\frac{1}{2}\left(\left.\phi\right|_{E^{l}}\right)\right|_{e} .
$$

If $e$ is adjacent to $\partial \Omega$, then $\boldsymbol{n}_{e}$ is the unit normal $\boldsymbol{n}$ exterior to $\Omega$ and the jump and the average of $\phi$ on $e$ coincide with the trace of $\phi$ on $e$. If $e$ is a segment of $\gamma_{h}^{1}$, we denote by $\boldsymbol{n}_{1}$ the outward normal to $\Omega_{1}$ and we set

$$
[\phi]=\left.\left(\left.\phi\right|_{\Omega_{1}}\right)\right|_{e}-\left.\left(\left.\phi\right|_{\Omega_{2}}\right)\right|_{e}, \quad\{\phi\}=\left.\frac{1}{2}\left(\left.\phi\right|_{\Omega_{1}}\right)\right|_{e}+\left.\frac{1}{2}\left(\left.\phi\right|_{\Omega_{2}}\right)\right|_{e}
$$

Then, we introduce the following bilinear forms on $\boldsymbol{X} \times \boldsymbol{X}$ and $\boldsymbol{X} \times M$, respectively:

$$
\begin{aligned}
a(\boldsymbol{u}, \boldsymbol{v})= & \sum_{E \in \mathcal{E}_{h}} \int_{E} \nabla \boldsymbol{u}: \nabla \boldsymbol{v} \\
& -\sum_{e \in \Gamma_{h} \cup \gamma_{h}^{1}} \int_{e}\{\nabla \boldsymbol{u}\} \boldsymbol{n}_{e} \cdot[\boldsymbol{v}]+\epsilon^{*} \sum_{e \in \Gamma_{h} \cup \gamma_{h}^{1}} \int_{e}\{\nabla \boldsymbol{v}\} \boldsymbol{n}_{e} \cdot[\boldsymbol{u}], \\
b(\boldsymbol{v}, p)= & -\sum_{E \in \mathcal{E}_{h}} \int_{E} p \nabla \cdot \boldsymbol{v}+\sum_{e \in \Gamma_{h}} \int_{e}\{p\}[\boldsymbol{v}] \cdot \boldsymbol{n}_{e}+\sum_{e \in \gamma_{h}^{1}} \int_{e} p_{\gamma}[\boldsymbol{v}] \cdot \boldsymbol{n}_{1},
\end{aligned}
$$

where $p_{\gamma}$ is the trace of $p$ on the coarser mesh, i.e.,

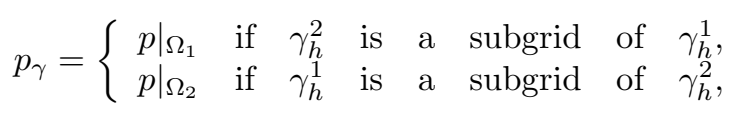

and on each segment $e$ of $\gamma_{h}^{1}$, the contribution of $\left.\nabla \boldsymbol{u}\right|_{\Omega_{2}}$ and $\left.\nabla \boldsymbol{v}\right|_{\Omega_{2}}$ stands for the piecewise gradient on each triangle of $\mathcal{E}_{h}^{2}$ adjacent to $e$. The parameter $\epsilon^{*}$ takes the constant value +1 or -1 . We also introduce the jump term

$$
J_{0}(\boldsymbol{u}, \boldsymbol{v})=\sum_{e \in \Gamma_{h} \cup \gamma_{h}^{1}} \frac{\sigma_{e}}{|e|} \int_{e}[\boldsymbol{u}] \cdot[\boldsymbol{v}],
$$

where $|e|$ denotes the measure of $e$. We assume throughout the paper one of the following cases:

(Ca) If the parameter $\epsilon^{*}=1$, then the jump coefficient can be simply equated to 1 on all edges. In practice, it could be useful to put more weight on some edges, but it is sufficient for the analysis to have $\sigma_{e} \geq \sigma_{0}$, independent of $h$.

(Cb) If the parameter $\epsilon^{*}=-1$, then the jump coefficient cannot be arbitrary. On one hand, it must be bounded below by $\sigma_{0}>0$ and above by $\sigma_{m}$. The analysis will show that it has to be sufficiently large. But in practice, its precise choice is delicate: if it is too large, the matrix of the discrete system will be ill-conditioned. 
Remark 3.1. The form $b$ defined by (3.3) can also be written

$$
b(\boldsymbol{v}, p)=-\sum_{E \in \mathcal{E}_{h}} \int_{E} p \nabla \cdot \boldsymbol{v}+\sum_{e \in \Gamma_{h} \cup \gamma_{h}^{1}} \int_{e}\{p\}[\boldsymbol{v}] \cdot \boldsymbol{n}_{e}+\frac{\epsilon}{2} \sum_{e \in \gamma_{h}^{1}} \int_{e}[p][\boldsymbol{v}] \cdot \boldsymbol{n}_{1},
$$

where

$$
\epsilon=\left\{\begin{array}{cccc}
1 & \text { if } \gamma_{h}^{2} \text { is a subgrid of } \gamma_{h}^{1}, \\
-1 & \text { if } \gamma_{h}^{1} \text { is a subgrid of } \gamma_{h}^{2} .
\end{array}\right.
$$

The extra jump term on $\gamma$ is added so that all jump terms on $\gamma$ disappear when one subdivision is a subset of the other. This is used for proving the inf-sup condition while doing domain decomposition.

Remark 3.2. Note that in (3.2), (3.3) and (3.4) each edge of $\mathcal{E}_{h}$ is counted exactly once. We have chosen the mesh $\gamma_{h}^{1}$ on the interface $\gamma$, but this choice is arbitrary and we could have chosen $\gamma_{h}^{2}$, in which case the jumps should be defined from $\Omega_{2}$ to $\Omega_{1}$.

Remark 3.3. Note that if $\boldsymbol{u}$ and $\boldsymbol{v}$ both belong to $H_{0}^{1}(\Omega)^{2}$, then formally

$$
a(\boldsymbol{u}, \boldsymbol{v})=\int_{\Omega} \nabla \boldsymbol{u}: \nabla \boldsymbol{v} \quad \text { and } \quad b(\boldsymbol{v}, p)=-\int_{\Omega} p \nabla \cdot \boldsymbol{v},
$$

which are the standard bilinear forms associated with the Stokes problem.

With these forms, we consider the following variational problem: Find $\boldsymbol{u} \in \boldsymbol{X}$ and $p \in M$, solution of

$$
\begin{aligned}
\mu\left(a(\boldsymbol{u}, \boldsymbol{v})+J_{0}(\boldsymbol{u}, \boldsymbol{v})\right)+b(\boldsymbol{v}, p) & =\int_{\Omega} \boldsymbol{f} \cdot \boldsymbol{v}, \quad \forall \boldsymbol{v} \in \boldsymbol{X}, \\
b(\boldsymbol{u}, q) & =0, \quad \forall q \in M .
\end{aligned}
$$

Remark 3.4. Note that all functions $\boldsymbol{v}$ in $\boldsymbol{X}$ satisfy

$$
-\sum_{E \in \mathcal{E}_{h}} \int_{E} \nabla \cdot \boldsymbol{v}+\sum_{e \in \Gamma_{h} \cup \gamma_{h}^{1}} \int_{e}[\boldsymbol{v}] \cdot \boldsymbol{n}_{e}=0 .
$$

Therefore we can relax the zero mean-value constraint in (3.6); i.e., (3.6) is equivalent to

$$
b(\boldsymbol{u}, q)=0, \quad \forall q \in Q=\left\{q \in L^{2}(\Omega): \forall E \in \mathcal{E}_{h},\left.q\right|_{E} \in W^{1,4 / 3}(E)\right\} .
$$

Remark 3.5. The case $\epsilon^{*}=1$ yields a nonsymmetric bilinear form $a$ and corresponds to the generalization of the NIPG method 23] whereas the case $\epsilon^{*}=-1$ yields a symmetric bilinear form $a$ and is the generalization of the SIPG method 27].

We denote by $\boldsymbol{V}$ the kernel of $b$ in $\boldsymbol{X}$ :

$$
\boldsymbol{V}=\{\boldsymbol{v} \in \boldsymbol{X} ; \quad \forall q \in M, \quad b(\boldsymbol{v}, q)=0\} .
$$

Lemma 3.6. Let $\boldsymbol{f} \in L^{4 / 3}(\Omega)^{2}$. If $(\boldsymbol{u}, p)$ is the solution of (2.1)-(2.3), then $(\boldsymbol{u}, p)$ satisfies the variational problem (3.5), (3.6) and conversely.

Proof. Let $(\boldsymbol{u}, p)$ be the solution of (2.1)-(2.3). Then $\nabla \cdot \boldsymbol{u}=0$ and $[\boldsymbol{u}] \cdot \boldsymbol{n}_{e}=0$ on each edge $e$, since $\boldsymbol{u} \in H_{0}^{1}(\Omega)^{2}$. Therefore, $\boldsymbol{u}$ satisfies (3.6). Let us multiply 
(2.1) by $\boldsymbol{v} \in \boldsymbol{X}$ and apply Green's formula in each $E$. As $\boldsymbol{u} \in W^{2,4 / 3}(\Omega)^{2}$ and $p \in W^{1,4 / 3}(\Omega)$, we have

$$
\begin{aligned}
\int_{\Omega}(-\mu \Delta \boldsymbol{u}+\nabla p) \cdot \boldsymbol{v} & =\sum_{E} \int_{E}(-\mu \Delta \boldsymbol{u}+\nabla p) \cdot \boldsymbol{v} \\
& =\sum_{E}\left(\int_{E}(\mu \nabla \boldsymbol{u}: \nabla \boldsymbol{v}-p \nabla \cdot \boldsymbol{v})-\int_{\partial E}\left(\mu \nabla \boldsymbol{u} \boldsymbol{n}_{E}-p \boldsymbol{n}_{E}\right) \cdot \boldsymbol{v}\right),
\end{aligned}
$$

where $\boldsymbol{n}_{E}$ is the outward normal to $\partial E$. But since $\mu \nabla \boldsymbol{u} \boldsymbol{n}_{e}-p \boldsymbol{n}_{e}$ are continuous across each interior edge, we have

$$
\left.\left(\mu \nabla \boldsymbol{u} \boldsymbol{n}_{e}-p \boldsymbol{n}_{e}\right)\right|_{e}=\left.\left\{\mu \nabla \boldsymbol{u} \boldsymbol{n}_{e}-p \boldsymbol{n}_{e}\right\}\right|_{e}=\left.\{\mu \nabla \boldsymbol{u}\}\right|_{e} \boldsymbol{n}_{e}-\left.\{p\}\right|_{e} \boldsymbol{n}_{e} .
$$

Therefore,

$$
\begin{aligned}
\int_{\Omega} \boldsymbol{f} \cdot \boldsymbol{v}= & \sum_{E} \int_{E}(\mu \nabla \boldsymbol{u}: \nabla \boldsymbol{v}-p \nabla \cdot \boldsymbol{v}) \\
& -\sum_{e \in \Gamma_{h} \cup \gamma_{h}^{1}} \int_{e}\{\mu \nabla \boldsymbol{u}\} \boldsymbol{n}_{e} \cdot[\boldsymbol{v}]+\sum_{e \in \Gamma_{h} \cup \gamma_{h}^{1}} \int_{e}\{p\}[\boldsymbol{v}] \cdot \boldsymbol{n}_{e} .
\end{aligned}
$$

This coincides with (3.5) because the jump of $\boldsymbol{u}$ is zero on each interior edge $e$, the jump of $p$ is zero on each edge $e$ of $\gamma_{h}^{1}$ and $\boldsymbol{u}$ vanishes on each boundary edge $e$.

Conversely, let $(\boldsymbol{u}, p)$ be a solution to (3.5), (3.6). First, let $E$ belong to $\mathcal{E}_{h}$ and choose $\boldsymbol{v} \in \mathcal{D}(E)^{2}$, extended by zero outside $E$. Then, $(\boldsymbol{u}, p)$ satisfies in the sense of distributions

$$
-\mu \Delta \boldsymbol{u}+\nabla p=\boldsymbol{f}, \quad \nabla \cdot \boldsymbol{u}=0, \quad \text { in } \quad E .
$$

Next consider $\boldsymbol{v} \in \mathcal{C}^{1}(\bar{E})^{2}$ such that $\boldsymbol{v}=\mathbf{0}$ on $\partial E$, extended by $\mathbf{0}$ outside $E$, and $\nabla \boldsymbol{v} \cdot \boldsymbol{n}_{E}=0$ on $\partial E$ except on one side $e$. We multiply (3.7) by $\boldsymbol{v}$ and integrate by parts:

$$
\mu \int_{E} \nabla \boldsymbol{u}: \nabla \boldsymbol{v}-\int_{E} p \nabla \cdot \boldsymbol{v}=\mu \int_{E} \nabla \boldsymbol{u}: \nabla \boldsymbol{v}-\int_{E} p \nabla \cdot \boldsymbol{v}+\mu \epsilon^{*} \int_{e}\{\nabla \boldsymbol{v}\} \boldsymbol{n}_{e} \cdot[\boldsymbol{u}],
$$

which is equivalent to

$$
\int_{e}\{\nabla \boldsymbol{v}\} \boldsymbol{n}_{e} \cdot[\boldsymbol{u}]=0
$$

Since $\{\nabla \boldsymbol{v}\} \boldsymbol{n}_{e}$ is arbitrary, $[\boldsymbol{u}]=\mathbf{0}$. If $e$ belongs to the boundary $\partial \Omega$, this implies that $\left.\boldsymbol{u}\right|_{\Omega}=\mathbf{0}$. Thus $\boldsymbol{u} \in H_{0}^{1}(\Omega)^{2}$. Finally, let $\boldsymbol{v} \in \mathcal{C}^{1}(\bar{E})^{2}$, with $\boldsymbol{v}=\mathbf{0}$ on $\partial E$ except on one side $e$, extended by $\mathbf{0}$ outside. First, we assume that $e$ does not belong to the interface, i.e., $e \in \Gamma_{h}$. We have

$$
\begin{gathered}
\mu \int_{E} \nabla \boldsymbol{u}: \nabla \boldsymbol{v}-\int_{E} p \nabla \cdot \boldsymbol{v}-\mu \int_{e} \nabla \boldsymbol{u} \boldsymbol{n}_{E} \cdot \boldsymbol{v}+\int_{e} p \boldsymbol{v} \cdot \boldsymbol{n}_{E} \\
=\mu \int_{E} \nabla \boldsymbol{u}: \nabla \boldsymbol{v}-\int_{E} p \nabla \cdot \boldsymbol{v}-\mu \int_{e}\{\nabla \boldsymbol{u}\} \boldsymbol{n}_{e} \cdot[\boldsymbol{v}]+\int_{e}\{p\}[\boldsymbol{v}] \cdot \boldsymbol{n}_{e},
\end{gathered}
$$

which implies

$$
\int_{e}\left(-\mu \nabla \boldsymbol{u} \boldsymbol{n}_{E}+p \boldsymbol{n}_{E}\right) \cdot \boldsymbol{v}=\int_{e}\left\{-\mu \nabla \boldsymbol{u} \boldsymbol{n}_{E}+p \boldsymbol{n}_{E}\right\} \cdot \boldsymbol{v} .
$$

Since $\boldsymbol{v}$ is arbitrary, this means that the quantity $-\mu \nabla \boldsymbol{u} \boldsymbol{n}_{E}+p \boldsymbol{n}_{E}$ is continuous across $e$. Therefore, $-\mu \Delta \boldsymbol{u}+\nabla p=\boldsymbol{f}$ in both subdomains $\Omega_{1}$ and $\Omega_{2}$. Next, let $e$ 
belong to the interface $\gamma_{h}^{1}$, and assume that $\gamma_{h}^{1}$ is a subgrid of $\gamma_{h}^{2}$. The case where $\gamma_{h}^{2}$ is a subgrid of $\gamma_{h}^{1}$ is handled exactly the same way. If $E$ belongs to $\mathcal{E}_{h}^{1}$, we have

$$
-\mu \int_{e} \nabla \boldsymbol{u} \boldsymbol{n}_{E} \cdot \boldsymbol{v}+\left.\int_{e} p\right|_{\Omega_{1}} \boldsymbol{v} \cdot \boldsymbol{n}_{E}=-\mu \int_{e}\{\nabla \boldsymbol{u}\} \boldsymbol{n}_{E} \cdot \boldsymbol{v}+\left.\int_{e} p\right|_{\Omega_{1}} \boldsymbol{v} \cdot \boldsymbol{n}_{E}
$$

therefore $\nabla \boldsymbol{u} \boldsymbol{n}_{E}$ is continuous across $e$. Similarly, if $E$ belongs to $\mathcal{E}_{h}^{2}$, we have

$$
\left.\int_{e} p\right|_{\Omega_{2}} \boldsymbol{v} \cdot \boldsymbol{n}_{E}=\left.\int_{e} p\right|_{\Omega_{1}} \boldsymbol{v} \cdot \boldsymbol{n}_{E}
$$

which implies that the jump of $p$ across $e$ is zero. Thus, $-\mu \Delta \boldsymbol{u}+\nabla p=\boldsymbol{f}$ in $\Omega$.

Remark 3.7. Note that the jump term $J_{0}$ plays no part in this proof and therefore the statement of Lemma 3.6 is valid even if $J_{0}$ is suppressed from (3.5).

In order to approximate $\boldsymbol{u}$ and $p$, we introduce two finite-dimensional spaces $\boldsymbol{X}_{h} \subset \boldsymbol{X}$ and $M_{h} \subset M$, such that

$$
\begin{aligned}
& X_{h}=\left\{v_{h} \in X: \quad \forall E \in \mathcal{E}_{h}, \quad v_{h} \in \mathbb{P}_{k}(E)\right\}, \quad \boldsymbol{X}_{h}=X_{h} \times X_{h}, \\
& M_{h}=\left\{q_{h} \in M: \quad \forall E \in \mathcal{E}_{h}, \quad q_{h} \in \mathbb{P}_{k-1}(E)\right\} .
\end{aligned}
$$

With these spaces, the discrete scheme is: find $(\boldsymbol{U}, P) \in \boldsymbol{X}_{h} \times M_{h}$ such that

$$
\begin{aligned}
\forall \boldsymbol{v}_{h} \in \boldsymbol{X}_{h}, \quad \mu\left(a\left(\boldsymbol{U}, \boldsymbol{v}_{h}\right)+J_{0}\left(\boldsymbol{U}, \boldsymbol{v}_{h}\right)\right)+b\left(\boldsymbol{v}_{h}, P\right) & =\int_{\Omega} \boldsymbol{f} \cdot \boldsymbol{v}_{h}, \\
\forall q_{h} \in M_{h}, \quad b\left(\boldsymbol{U}, q_{h}\right) & =0 .
\end{aligned}
$$

We denote by $\boldsymbol{V}_{h}$ the kernel of $b$ in $\boldsymbol{X}_{h}$ :

$$
\boldsymbol{V}_{h}=\left\{\boldsymbol{v}_{h} \in \boldsymbol{X}_{h} ; \quad \forall q_{h} \in M_{h}, \quad b\left(\boldsymbol{v}_{h}, q_{h}\right)=0\right\},
$$

and we observe that, as in the continuous case, we can relax the zero mean-value constraint in (3.9). We shall address in the next section the existence and uniqueness of the solution.

Remark 3.8. If $\boldsymbol{U} \in \boldsymbol{X}_{h}$ satisfies (3.9), i.e., $\boldsymbol{U} \in \boldsymbol{V}_{h}$, then for all $E \in \mathcal{E}_{h}$, not adjacent to $\gamma$

$$
\int_{\partial E}\{\boldsymbol{U}\} \cdot \boldsymbol{n}_{E}=0
$$

but if $E$ is adjacent to $\gamma$ and $e$ is the side of $E$ on $\gamma$, we have

$$
\int_{\partial E}\{\boldsymbol{U}\} \cdot \boldsymbol{n}_{E}-\frac{\epsilon}{2} \int_{e}[\boldsymbol{U}] \cdot \boldsymbol{n}_{E}=0
$$

Thus the discrete mass is conserved on each triangle $E$ that is not adjacent to the interface. As mentionned in the introduction, the factor of $\epsilon$ in the definition of $b$ is added to prove the inf-sup condition. Indeed, if there is no domain decomposition, i.e., no interface, then the analysis goes through with the following $b$, which is what one expects:

$$
b(\boldsymbol{v}, p)=-\sum_{E \in \mathcal{E}_{h}} \int_{E} p \nabla \cdot \boldsymbol{v}+\sum_{e \in \Gamma_{h}} \int_{e}\{p\}[\boldsymbol{v}] \cdot \boldsymbol{n}_{e} .
$$

In this case, the mass is conserved on all elements. 
Finally, let us recall the approximation properties of $\boldsymbol{X}_{h}$ and $M_{h}$. Recall that the meshes $\mathcal{E}_{h}^{i}$ are regular (see (3.1)). For each integer $k \geq 1$, it is easy to construct an operator $r_{h} \in \mathcal{L}\left(L_{0}^{2}(\Omega) ; M_{h}\right)$, such that, for any $E \in \mathcal{E}_{h}$,

$$
\forall q \in \mathbb{P}_{k-1}(E), \quad \int_{E} q\left(r_{h}(p)-p\right)=0,
$$

and for any real number $s \in[0, k]$,

$$
\forall q \in H^{s}(\Omega) \cap L_{0}^{2}(\Omega), \quad\left\|q-r_{h}(q)\right\|_{0, E} \leq C h_{E}^{s}|q|_{s, E} .
$$

For each $k=1,2,3$, there exists an operator $\boldsymbol{R}_{h}^{i} \in \mathcal{L}\left(H^{1}\left(\Omega_{i}\right)^{2} ; \boldsymbol{X}_{h}\left(\Omega_{i}\right)\right)$, where $\boldsymbol{X}_{h}\left(\Omega_{i}\right)$ denotes the space $\boldsymbol{X}_{h}$ restricted to $\Omega_{i}$, such that for any $E \in \mathcal{E}_{h}$,

$$
\forall \boldsymbol{v} \in H^{1}\left(\Omega_{i}\right)^{2}, \quad \forall \text { interior } \quad e \quad \text { of } \quad \Gamma_{h}^{i}, \quad \forall \boldsymbol{q}_{h} \in \mathbb{P}_{k-1}(e)^{2}, \quad \int_{e} \boldsymbol{q}_{h} \cdot\left[\boldsymbol{R}_{h}^{i}(\boldsymbol{v})\right]=0,
$$

$$
\begin{aligned}
& \forall \boldsymbol{v} \in H_{0}^{1}\left(\Omega_{i}\right)^{2}, \quad \forall e \in \partial \Omega_{i}, \quad \forall \boldsymbol{q}_{h} \in \mathbb{P}_{k-1}(e)^{2}, \quad \int_{e} \boldsymbol{q}_{h} \cdot \boldsymbol{R}_{h}^{i}(\boldsymbol{v})=0, \\
& \forall s \in[1, k+1], \quad \forall \boldsymbol{v} \in H^{s}\left(\Omega_{i}\right)^{2}, \quad\left|\boldsymbol{v}-\boldsymbol{R}_{h}^{i}(\boldsymbol{v})\right|_{1, E} \leq C h_{E}^{s-1}|\boldsymbol{v}|_{s, \Delta_{E}},
\end{aligned}
$$

where $\Delta_{E}$ is a suitable macro-element containing $E$. When $k=1, \Delta_{E}=E$. The case $k=1$ follows from [7], $k=2$ from [9] and $k=3$ from [6]. It also follows from Girault and Scott [13 that, for $m=0$ or 1 , for any $t \geq 2$, for $s \in[1, k+1]$,

$$
\forall \boldsymbol{v} \in W^{s, t}\left(\Omega_{i}\right)^{2}, \quad\left|\boldsymbol{v}-\boldsymbol{R}_{h}^{i}(\boldsymbol{v})\right|_{W^{m, t}(E)} \leq C h_{E}^{s-m}|\boldsymbol{v}|_{W^{s, t}\left(\Delta_{E}\right)} .
$$

Furthermore, each triangle $E \in E_{h}^{i}$ has at least one side $e$ such that

$$
\forall \boldsymbol{v} \in H^{1}\left(\Omega_{i}\right)^{2}, \quad \int_{e}\left(\boldsymbol{R}_{h}^{i}(\boldsymbol{v})-\boldsymbol{v}\right)=\mathbf{0} .
$$

This property is obvious when $k=1$ and it holds on all edges $e$ of $\Gamma_{h}^{i} \cup \gamma_{h}^{i}$, because in this case there is only one degree of freedom per edge and it is defined by $|e|^{-1} \int_{e} v$. When $k=2$, 3.17) also holds on all edges $e$ of $\Gamma_{h}^{i} \cup \gamma_{h}^{i}$, because it is a consequence of formulas (19) and (49) of reference [9]. When $k=3$, for most practical meshes, it holds on all edges $e$ of $\Gamma_{h}^{i} \cup \gamma_{h}^{i}$, with the exception of the interior edges of the configuration on Figure 2 But as the boundary edges of this configuration satisfy

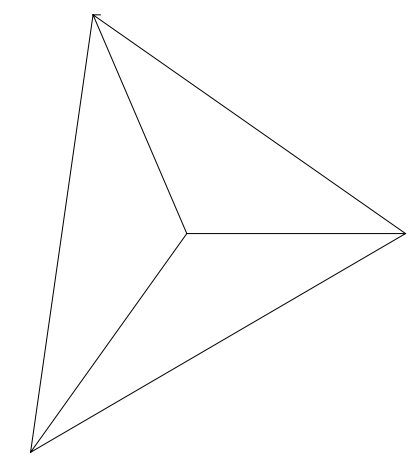

Figure 2. Special configuration for $k=3$. 
(3.17), each triangle in this configuration has an edge where (3.17) holds. An easy consequence of (3.17) is the following lemma.

Lemma 3.9. Assume that $\mathcal{E}_{h}^{i}$ satisfies (3.1). Then there exists a constant $C$ independent of $h$, such that

$$
\forall \boldsymbol{v} \in\left(H_{0}^{1}\left(\Omega_{i}\right)\right)^{2}, \quad\left(\sum_{e \in \Gamma_{h}^{i}} \frac{1}{|e|}\left\|\left[\boldsymbol{v}-\boldsymbol{R}_{h}^{i}(\boldsymbol{v})\right]\right\|_{0, e}^{2}\right)^{1 / 2} \leq C\left\|\nabla\left(\boldsymbol{v}-\boldsymbol{R}_{h}^{i}(\boldsymbol{v})\right)\right\|_{0, \Omega_{i}} .
$$

\section{AN INF-SUP CONDITION}

For proving an inf-sup condition, we must choose a norm on $\boldsymbol{X}$ that is more appropriate than the broken $H^{1}$ norm. In the exact case, $X=H_{0}^{1}(\Omega)$ and the intrinsic norm is the $H^{1}$ seminorm. But clearly, this is not a norm on $\boldsymbol{X}_{h}$ and completing this seminorm with the $L^{2}$ norm does not represent sufficiently well the jumps on the edges. Therefore, we propose to complete the seminorm with the jump term $J_{0}$. This idea is not new, it has already been used by Wheeler in [27. Thus, we define the norm

$$
\forall \boldsymbol{v}_{h} \in \boldsymbol{X}_{h}, \quad \llbracket \boldsymbol{v}_{h} \rrbracket=\left(\sum_{E \in \mathcal{E}_{h}}\left\|\nabla \boldsymbol{v}_{h}\right\|_{0, E}^{2}+J_{0}\left(\boldsymbol{v}_{h}, \boldsymbol{v}_{h}\right)\right)^{1 / 2} .
$$

If there is no domain decomposition, the inf-sup condition follows directly from the properties of $\boldsymbol{R}_{h}$ stated in Section 3. But a special proof is required if there is domain decomposition with nonmatching grids. In this case, we need the following assumptions.

Hypothesis H1: Either $\gamma_{h}^{1}$ is a subgrid of $\gamma_{h}^{2}$ or $\gamma_{h}^{2}$ is a subgrid of $\gamma_{h}^{1}$.

Hypothesis H2: There exist two constants $L_{1}$ and $L_{2}$ independent of $h$ such that for any pair of segments $e_{1} \in \gamma_{h}^{1}$ and $e_{2} \in \gamma_{h}^{2}$ such that $\left|e_{1} \cap e_{2}\right|>0$, we have

$$
\frac{\left|e_{1}\right|}{\left|e_{2}\right|} \leq L_{1} \quad \text { and } \quad \frac{\left|e_{2}\right|}{\left|e_{1}\right|} \leq L_{2} \text {. }
$$

Remark 4.1. Since by assumption, $\gamma_{h}^{1}$ is a subgrid of $\gamma_{h}^{2}$ or vice versa, then one of the two constants $L_{1}$ or $L_{2}$ is one; but this precise value is not used here.

Then, we have the following preliminary result.

Lemma 4.2. Let the mesh $\mathcal{E}_{h}$ satisfy (3.1) and (4.2). Let $\boldsymbol{v} \in H^{1}(\Omega)^{2}$, let $k=1,2$ or 3 and let $\boldsymbol{R}_{h}(\boldsymbol{v})$ denote the operators $\boldsymbol{R}_{h}^{1}(\boldsymbol{v})$ in $\Omega_{1}$ and $\boldsymbol{R}_{h}^{2}(\boldsymbol{v})$ in $\Omega_{2}$ satisfying (3.13) -(3.17). Then there exists a constant $C$ that depends only on $k, L_{1}, L_{2}$ and the constant $\sigma$ of (3.1) such that

$$
\forall p_{h} \in M_{h}, \quad\left|\sum_{e \in \Gamma_{h} \cup \gamma_{h}^{1}} \int_{e}\left\{p_{h}\right\}\left[\boldsymbol{R}_{h}(\boldsymbol{v})-\boldsymbol{v}\right] \cdot \boldsymbol{n}_{e}\right| \leq C\left\|p_{h}\right\|_{0, D^{12}}\left\|\nabla\left(\boldsymbol{R}_{h}(\boldsymbol{v})-\boldsymbol{v}\right)\right\|_{0, D^{12}},
$$

where $D^{12}=D_{1} \cup D_{2}$ and $D_{i}$ denotes the union of elements of $\mathcal{E}_{h}^{i}$ adjacent to $\gamma$.

Proof. Consider

$$
\left|\sum_{e \in \gamma_{h}^{1}} \int_{e}\left\{p_{h}\right\}\left[\boldsymbol{R}_{h}(\boldsymbol{v})-\boldsymbol{v}\right] \cdot \boldsymbol{n}_{e}\right| \leq \int_{\gamma}\left|\left\{p_{h}\right\}\left[\boldsymbol{R}_{h}(\boldsymbol{v})-\boldsymbol{v}\right] \cdot \boldsymbol{n}_{1}\right|,
$$


since the contribution of the left-hand side of (4.3) to the edges $e$ of $\Gamma_{h}$ is zero by virtue of (3.13). Expanding $\left\{p_{h}\right\}$ and $\left[\boldsymbol{R}_{h}(\boldsymbol{v})-\boldsymbol{v}\right] \cdot \boldsymbol{n}_{1}$, we can write

$$
\begin{aligned}
& \int_{\gamma}\left|\left\{p_{h}\right\}\left[\boldsymbol{R}_{h}(\boldsymbol{v})-\boldsymbol{v}\right] \cdot \boldsymbol{n}_{1}\right| \\
& \leq \frac{1}{2}\left(\left.\int_{\gamma}\left|p_{h}\right| \Omega_{1}\left|\left\|\left.\left(\boldsymbol{R}_{h}(\boldsymbol{v})-\boldsymbol{v}\right)\right|_{\Omega_{1}}\right\|+\int_{\gamma}\right| p_{h}\left|\Omega_{\Omega_{1}}\right|||\left(\boldsymbol{R}_{h}(\boldsymbol{v})-\boldsymbol{v}\right)\right|_{\Omega_{2}} \|\right. \\
& \quad+\left.\int_{\gamma}\left|p_{h}\right| \Omega_{\Omega_{2}}||\left(\boldsymbol{R}_{h}(\boldsymbol{v})-\boldsymbol{v}\right)\right|_{\Omega_{1}}\left\|+\int_{\gamma}\left|p_{h}\right| \Omega_{\Omega_{2}}\left|\left\|\left.\left(\boldsymbol{R}_{h}(\boldsymbol{v})-\boldsymbol{v}\right)\right|_{\Omega_{2}}\right\|\right)\right.
\end{aligned}
$$

where $\|\cdot\|$ denotes here the Euclidean norm. To bound the first integral, let $e \in \gamma_{h}^{1}$ and let $E \in \mathcal{E}_{h}^{1}$ be adjacent to $e$; we have

$$
\begin{aligned}
\int_{e}\left|p_{h}\right|_{\Omega_{1}}\left|\left\|\left.\left(\boldsymbol{R}_{h}(\boldsymbol{v})-\boldsymbol{v}\right)\right|_{\Omega_{1}}\right\|\right. & \leq\left\|\left.p_{h}\right|_{\Omega_{1}}\right\|_{0, e}\left\|\left.\left(\boldsymbol{R}_{h}(\boldsymbol{v})-\boldsymbol{v}\right)\right|_{\Omega_{1}}\right\|_{0, e} \\
& \leq \hat{C}|e||E|^{-1 / 2}\left\|p_{h}\right\|_{0, E}\left\|\widehat{\boldsymbol{R}_{h}(\boldsymbol{v})}-\hat{\boldsymbol{v}}\right\|_{0, \hat{e}}
\end{aligned}
$$

because $\hat{p}_{h}$ belongs to a finite-dimensional space on $\hat{E}$. Here $\hat{C}$ denotes various constants independent of $h$. On one hand, by the trace theorem,

$$
\left\|\widehat{\boldsymbol{R}_{h}(\boldsymbol{v})}-\hat{\boldsymbol{v}}\right\|_{0, \hat{e}} \leq \hat{C}\left\|\widehat{\boldsymbol{R}_{h}(\boldsymbol{v})}-\hat{\boldsymbol{v}}\right\|_{1, \hat{E}} \text {. }
$$

On the other hand, $\hat{E}$ has one side on which (3.17) holds. Therefore, as in Lemma [3.9] there exists a constant $\hat{C}$ such that

$$
\left\|\widehat{\boldsymbol{R}_{h}(\boldsymbol{v})}-\hat{\boldsymbol{v}}\right\|_{0, \hat{e}} \leq \hat{C}\left|\widehat{\boldsymbol{R}_{h}(\boldsymbol{v})}-\hat{\boldsymbol{v}}\right|_{1, \hat{E}}
$$

Hence, reverting to $E$ and applying (3.1), we obtain

$$
\int_{e}\left|p_{h}\right|_{\Omega_{1}}\left|\left\|\left.\left(\boldsymbol{R}_{h}(\boldsymbol{v})-\boldsymbol{v}\right)\right|_{\Omega_{1}}\right\| \leq \hat{C}\left\|p_{h}\right\|_{0, E}\left\|\nabla\left(\boldsymbol{R}_{h}(\boldsymbol{v})-\boldsymbol{v}\right)\right\|_{0, E} .\right.
$$

Thus denoting by $D_{1}$ the row of elements of $\mathcal{E}_{h}^{1}$ adjacent to $\gamma$, we derive

$$
\int_{\gamma}\left|p_{h}\right|_{\Omega_{1}}\left|\left\|\left.\left(\boldsymbol{R}_{h}(\boldsymbol{v})-\boldsymbol{v}\right)\right|_{\Omega_{1}}\right\| \leq \hat{C}\left\|p_{h}\right\|_{0, D_{1}}\left\|\nabla\left(\boldsymbol{R}_{h}(\boldsymbol{v})-\boldsymbol{v}\right)\right\|_{0, D_{1}} .\right.
$$

Similarly, denoting by $D_{2}$ the row of elements of $\mathcal{E}_{h}^{2}$ adjacent to $\gamma$, we bound the fourth integral by

$$
\int_{\gamma}\left|p_{h}\right|_{\Omega_{2}}\left|\left\|\left.\left(\boldsymbol{R}_{h}(\boldsymbol{v})-\boldsymbol{v}\right)\right|_{\Omega_{2}}\right\| \leq \hat{C}\left\|p_{h}\right\|_{0, D_{2}}\left\|\nabla\left(\boldsymbol{R}_{h}(\boldsymbol{v})-\boldsymbol{v}\right)\right\|_{0, D_{2}}\right.
$$

To bound the second integral, consider again $e \in \gamma_{h}^{1}$; we can write

$$
\int_{e}\left|p_{h}\right|_{\Omega_{1}}\left|\left\|\left.\left(\boldsymbol{R}_{h}(\boldsymbol{v})-\boldsymbol{v}\right)\right|_{\Omega_{2}}\right\| \leq\left\|\left.p_{h}\right|_{\Omega_{1}}\right\|_{0, e}\left(\sum_{j}\left\|\boldsymbol{R}_{h}(\boldsymbol{v})-\boldsymbol{v}\right\|_{0, e_{j}}^{2}\right)^{1 / 2},\right.
$$

where $j$ runs over all segments $e_{j}$ of $\gamma_{h}^{2}$ that intersect $e$. Thus,

$$
\begin{aligned}
\int_{e}\left|p_{h}\right| \Omega_{1}\left|\left\|\left.\left(\boldsymbol{R}_{h}(\boldsymbol{v})-\boldsymbol{v}\right)\right|_{\Omega_{2}}\right\|\right. & \leq \hat{C}\left|e\left\|\left.E\right|^{-1 / 2}\right\| p_{h} \|_{0, E}\left(\sum_{j} \frac{\left|e_{j}\right|}{|e|}\left|\boldsymbol{R}_{h}(\boldsymbol{v})-\boldsymbol{v}\right|_{1, E_{j}}^{2}\right)^{1 / 2}\right. \\
& \leq \hat{C} L_{2}^{1 / 2}\left\|p_{h}\right\|_{0, E}\left\|\nabla\left(\boldsymbol{R}_{h}(\boldsymbol{v})-\boldsymbol{v}\right)\right\|_{0, \cup_{j} E_{j}},
\end{aligned}
$$


by virtue of (4.2), where $E_{j}$ denotes the element of $\mathcal{E}_{h}^{2}$ that is adjacent to $e_{j}$ and the union runs over all $e_{j}$ of $\gamma_{h}^{2}$ that intersect $e$. Hence

$$
\int_{\gamma}\left|p_{h}\right|_{\Omega_{1}}\left|\left\|\left.\left(\boldsymbol{R}_{h}(\boldsymbol{v})-\boldsymbol{v}\right)\right|_{\Omega_{2}}\right\| \leq \hat{C} L_{2}^{1 / 2}\left\|p_{h}\right\|_{0, D_{1}}\left\|\nabla\left(\boldsymbol{R}_{h}(\boldsymbol{v})-\boldsymbol{v}\right)\right\|_{0, D_{2}} .\right.
$$

Similarly, applying the first inequality in (4.2), the third integral is bounded by

$$
\int_{\gamma}\left|p_{h}\right|_{\Omega_{2}}\left|\left\|\left.\left(\boldsymbol{R}_{h}(\boldsymbol{v})-\boldsymbol{v}\right)\right|_{\Omega_{1}}\right\| \leq \hat{C} L_{1}^{1 / 2}\left\|p_{h}\right\|_{0, D_{2}}\left\|\nabla\left(\boldsymbol{R}_{h}(\boldsymbol{v})-\boldsymbol{v}\right)\right\|_{0, D_{1}} .\right.
$$

Collecting these bounds, we obtain

$$
\begin{aligned}
& \left|\int_{\gamma}\left\{p_{h}\right\}\left[\boldsymbol{R}_{h}(\boldsymbol{v})-\boldsymbol{v}\right] \cdot \boldsymbol{n}_{1}\right| \\
& \quad \leq \frac{1}{\sqrt{2}} \hat{C}\left(1+L_{1}+L_{2}\right)^{1 / 2}\left\|p_{h}\right\|_{0, D_{1} \cup D_{2}}\left\|\nabla\left(\boldsymbol{R}_{h}(\boldsymbol{v})-\boldsymbol{v}\right)\right\|_{0, D_{1} \cup D_{2}} .
\end{aligned}
$$

This proves (4.3).

Remark 4.3. More generally, if $\boldsymbol{v}_{h} \in \boldsymbol{X}_{h}$ satisfies only the analogue of (3.17), then (4.3) is replaced by

$$
\forall p_{h} \in M_{h}, \quad\left|\sum_{e \in \Gamma_{h} \cup \gamma_{h}^{1}} \int_{e}\left\{p_{h}\right\}\left[\boldsymbol{v}_{h}-\boldsymbol{v}\right] \cdot \boldsymbol{n}_{e}\right| \leq C\left\|p_{h}\right\|_{0, \Omega}\left\|\nabla\left(\boldsymbol{v}_{h}-\boldsymbol{v}\right)\right\|_{0} .
$$

Indeed, the jumps on the interior edges of $\Gamma_{h}$ vanish when (3.13) holds. If we only have (3.17), then

$$
\left|\sum_{e \in \Gamma_{h}} \int_{e}\left\{p_{h}\right\}\left[\boldsymbol{v}_{h}-\boldsymbol{v}\right] \cdot \boldsymbol{n}_{e}\right| \leq C\left\|p_{h}\right\|_{0, \Omega}\left\|\nabla\left(\boldsymbol{v}_{h}-\boldsymbol{v}\right)\right\|_{0} .
$$

Remark 4.4. Similarly,

$$
\left|\sum_{e \in \gamma_{h}^{1}} \int_{e}\left[p_{h}\right]\left[\boldsymbol{R}_{h}(\boldsymbol{v})-\boldsymbol{v}\right] \cdot \boldsymbol{n}_{e}\right| \leq C\left\|p_{h}\right\|_{0, D^{12}}\left\|\nabla\left(\boldsymbol{R}_{h}(\boldsymbol{v})-\boldsymbol{v}\right)\right\|_{0, D^{12}} .
$$

Now we can address the existence and uniqueness of (3.8), (3.9). Since it is a square system of linear equations in finite dimension, it suffices to prove that $\boldsymbol{f}=\mathbf{0}$ implies $\boldsymbol{U}=\mathbf{0}$ and $P=0$. We choose $\boldsymbol{v}_{h}=\boldsymbol{U}$. In the nonsymmetric case, this implies that $a(\boldsymbol{U}, \boldsymbol{U})=0$ and $J_{0}(\boldsymbol{U}, \boldsymbol{U})=0$, and thus $\boldsymbol{U}=0$. In the symmetric case, we make the following assumption.

Hypothesis H3: There exists a constant $K>0$, independent of $h$, such that

$$
\forall \boldsymbol{v}_{h} \in \boldsymbol{X}_{h}, \quad a\left(\boldsymbol{v}_{h}, \boldsymbol{v}_{h}\right)+J_{0}\left(\boldsymbol{v}_{h}, \boldsymbol{v}_{h}\right) \geq K \llbracket \boldsymbol{v}_{h} \rrbracket^{2} .
$$

In the nonsymmetric case, $K=1$. In the symmetric case, a standard proof [27] shows that $\mathrm{H} 3$ holds if $\mathrm{H} 2$ is valid and the coefficients $\sigma_{e}$ in (3.4) are sufficiently large (but independent of $h$ ). Hence in both cases, $b\left(\boldsymbol{v}_{h}, P\right)=0$ for all $\boldsymbol{v}_{h}$ in $\boldsymbol{X}_{h}$. We shall see that $P=0$ by virtue of Theorem 4.5 .

So far, we have not used the fact that $\gamma_{h}^{1}$ is a subgrid of $\gamma_{h}^{2}$ (or vice versa). But now we shall use it for proving the inf-sup condition, so we make this assumption from now on. 
Theorem 4.5. Let the mesh $\mathcal{E}_{h}$ satisfy (3.1) and Hypotheses $\mathrm{H} 1$ and H2. Then, there exists a constant $\beta^{*}>0$, independent of $h$, such that

$$
\inf _{p_{h} \in M_{h}} \sup _{\boldsymbol{v}_{h} \in \widetilde{\boldsymbol{X}}_{h}} \frac{b\left(\boldsymbol{v}_{h}, p_{h}\right)}{\llbracket \mid \boldsymbol{v}_{h} \rrbracket\left\|p_{h}\right\|_{0}} \geq \beta^{*},
$$

where

$$
\widetilde{\boldsymbol{X}_{h}}=\left\{\boldsymbol{v}_{h} \in \boldsymbol{X}_{h}: \forall e \in \Gamma_{h}, \quad \int_{e} \boldsymbol{q}_{h} \cdot\left[\boldsymbol{v}_{h}\right]=0, \quad \forall \boldsymbol{q}_{h} \in \mathbb{P}_{k-1}(e)^{2}\right\} .
$$

Proof. We shall prove that for any $p_{h}$ in $M_{h}$, there exists $\boldsymbol{v}_{h}$ in $\widetilde{\boldsymbol{X}_{h}}$ such that

$$
\begin{aligned}
b\left(\boldsymbol{v}_{h}, p_{h}\right) & \geq \beta_{1}^{*}\left\|p_{h}\right\|_{0}^{2}, \\
{\left[\boldsymbol{v}_{h}\right] } & \leq \beta_{2}^{*}\left\|p_{h}\right\|_{0},
\end{aligned}
$$

with constants $\beta_{1}^{*}>0$ and $\beta_{2}^{*}>0$ independent of $h, p_{h}$ and $\boldsymbol{v}_{h}$. Clearly, this will imply (4.8). Let $p_{h} \in M_{h}$. The idea of the proof is to use the inf-sup condition that holds separately in each $\Omega_{i}$ and to correct it suitably in order to account for the interface. For this, we split $p_{h}$ into two functions with zero mean value in $\Omega_{i}$ plus two constant functions in $\Omega_{i}$. Thus we define

$$
\bar{p}_{h}^{i}=\frac{1}{\left|\Omega_{i}\right|} \int_{\Omega_{i}} p_{h}, \quad \tilde{p}_{h}^{i}=\left.p_{h}\right|_{\Omega_{i}}-\bar{p}_{h}^{i},\left.\quad \tilde{p}_{h}\right|_{\Omega_{i}}=\tilde{p}_{h}^{i},\left.\quad \bar{p}_{h}\right|_{\Omega_{i}}=\bar{p}_{h}^{i} .
$$

Since $\tilde{p}_{h}^{i} \in L_{0}^{2}\left(\Omega_{i}\right)$ and the spaces $H_{0}^{1}\left(\Omega_{i}\right)^{2}, L_{0}^{2}\left(\Omega_{i}\right)$ satisfy the exact inf-sup condition (see for example [12]), there exists $\tilde{\boldsymbol{v}}^{i} \in H_{0}^{1}\left(\Omega_{i}\right)^{2}$ such that

$$
-\nabla \cdot \tilde{\boldsymbol{v}}_{i}=\tilde{p}_{h}^{i}, \quad\left|\tilde{\boldsymbol{v}}_{i}\right|_{1, \Omega_{i}} \leq \frac{1}{\beta}\left\|\tilde{p}_{h}^{i}\right\|_{0, \Omega_{i}} .
$$

Define $\tilde{\boldsymbol{v}}_{h}^{i}=\boldsymbol{R}_{h}^{i}\left(\tilde{\boldsymbol{v}}^{i}\right)$ and define $\tilde{\boldsymbol{v}}_{h}$ by $\left.\tilde{\boldsymbol{v}}_{h}\right|_{\Omega_{i}}=\tilde{\boldsymbol{v}}_{h}^{i}$. Then $\tilde{\boldsymbol{v}}_{h} \in \boldsymbol{X}_{h}$ and satisfies

$$
\begin{aligned}
-\sum_{E \in \mathcal{E}_{h}} \int_{E}\left(\nabla \cdot \tilde{\boldsymbol{v}}_{h}\right) \tilde{p}_{h} & =\int_{\Omega}\left(\tilde{p}_{h}\right)^{2}, \\
\left(\sum_{E \in \mathcal{E}_{h}}\left|\tilde{\boldsymbol{v}}_{h}\right|_{1, E}^{2}\right)^{1 / 2} \leq \frac{C_{1}}{\beta}\left\|\tilde{p}_{h}\right\|_{0, \Omega}, & \\
\forall e \in \Gamma_{h}, \quad \forall \boldsymbol{q} \in \mathbb{P}_{k-1}(e)^{2}, \quad \int_{e} \boldsymbol{q} \cdot\left[\tilde{\boldsymbol{v}}_{h}\right] & =0, \quad \text { i.e., } \quad \tilde{\boldsymbol{v}}_{h} \in \widetilde{\boldsymbol{X}_{h}} \\
\forall e \in \gamma_{h}^{i}, \quad \forall \boldsymbol{q} \in \mathbb{P}_{k-1}(e)^{2}, \quad \int_{e} \boldsymbol{q} \cdot \tilde{\boldsymbol{v}}_{h}^{i} & =0,
\end{aligned}
$$

with a constant $C_{1}$ independent of $h$ and $\beta$. To simplify, take $\epsilon=1$. The case $\epsilon=-1$ is treated similarly.

$$
b\left(\tilde{\boldsymbol{v}}_{h}, \tilde{p}_{h}\right)=\left\|\tilde{p}_{h}\right\|_{0}^{2}+\sum_{e \in \gamma_{h}^{1}} \int_{e} \tilde{p}_{h}^{1}\left(\boldsymbol{R}_{h}\left(\tilde{\boldsymbol{v}}^{1}\right)-\boldsymbol{R}_{h}\left(\tilde{\boldsymbol{v}}^{2}\right)\right) \cdot \boldsymbol{n}_{1} .
$$

(Recall that $\boldsymbol{n}_{1}$ is the normal on $\gamma$, exterior to $\Omega_{1}$.) But in view of (3.14),

$$
\int_{e} \tilde{p}_{h}^{1} \boldsymbol{R}_{h}\left(\tilde{\boldsymbol{v}}^{1}\right) \cdot \boldsymbol{n}_{1}=0 ;
$$


and since $\gamma_{h}^{2}$ is a subgrid of $\gamma_{h}^{1}$, then on each $e_{j}$ of $\gamma_{h}^{2}$ that is contained in $e, \tilde{p}_{h}^{1}$ is a polynomial of $\mathbb{P}_{k-1}$. Therefore (3.14) implies also that

$$
\sum_{j} \int_{e_{j}} \tilde{p}_{h}^{1} \boldsymbol{R}_{h}\left(\tilde{\boldsymbol{v}}^{2}\right) \cdot \boldsymbol{n}_{1}=0 .
$$

Note that this is the only instance where we use Hypothesis $\mathrm{H} 3$. Thus,

$$
b\left(\tilde{\boldsymbol{v}}_{h}, \tilde{p}_{h}\right)=\left\|\tilde{p}_{h}\right\|_{0}^{2}
$$

As far as the piecewise constant functions are concerned, note that $\bar{p}_{h} \in L_{0}^{2}(\Omega)$ because $p_{h} \in L_{0}^{2}(\Omega)$, i.e., $\bar{p}_{h}^{1}\left|\Omega_{1}\right|+\bar{p}_{h}^{2}\left|\Omega_{2}\right|=0$. Take $\overline{\boldsymbol{v}}=\alpha \boldsymbol{\rho}, \alpha$ to be chosen, where $\boldsymbol{\rho}$ is a function in $\mathcal{C}^{2}(\bar{\Omega})^{2}$, with compact support in $\Omega$ such that $\int_{\gamma} \boldsymbol{\rho} \cdot \boldsymbol{n}_{1}=1$. Let $\bar{q}_{h} \in L_{0}^{2}(\Omega)$ such that

$$
\bar{q}_{h}=\left\{\begin{array}{lllll}
\bar{q}_{h}^{1} & \text { in } & \Omega_{1}, & \bar{q}_{h}^{1} & \text { constant } \\
\bar{q}_{h}^{2} & \text { in } & \Omega_{2}, & \bar{q}_{h}^{2} & \text { constant. }
\end{array}\right.
$$

We want to find $\alpha$ so that for all $\bar{q}_{h}$ defined as above,

$$
-\int_{\Omega}(\nabla \cdot \overline{\boldsymbol{v}}) \bar{q}_{h}=\int_{\Omega} \bar{p}_{h} \bar{q}_{h}
$$

i.e.,

$$
-\int_{\Omega_{1}}(\nabla \cdot \overline{\boldsymbol{v}}) \bar{q}_{h}^{1}-\int_{\Omega_{2}}(\nabla \cdot \overline{\boldsymbol{v}}) \bar{q}_{h}^{2}=\left|\Omega_{1}\right| \bar{p}_{h}^{1} \bar{q}_{h}^{1}+\left|\Omega_{2}\right| \bar{p}_{h}^{2} \bar{q}_{h}^{2} .
$$

Applying Green's formula and using the continuity of $\overline{\boldsymbol{v}}$, this becomes

$$
-\left.\left(\bar{q}_{h}^{1}-\bar{q}_{h}^{2}\right) \int_{\gamma} \overline{\boldsymbol{v}}\right|_{\Omega_{1}} \cdot \boldsymbol{n}_{1}=\left|\Omega_{1}\right| \bar{p}_{h}^{1} \bar{q}_{h}^{1}+\left|\Omega_{2}\right| \bar{p}_{h}^{2} \bar{q}_{h}^{2} .
$$

As $\overline{\boldsymbol{v}}=\alpha \boldsymbol{\rho}$ and $\int_{\gamma} \boldsymbol{\rho} \cdot \boldsymbol{n}_{1}=1$, this holds if

$$
-\alpha\left(\bar{q}_{h}^{1}-\bar{q}_{h}^{2}\right)=\left|\Omega_{1}\right| \bar{p}_{h}^{1} \bar{q}_{h}^{1}+\left|\Omega_{2}\right| \bar{p}_{h}^{2} \bar{q}_{h}^{2} .
$$

Let us multiply this equation by $\left|\Omega_{2}\right|$ and use the fact that $\left|\Omega_{1}\right| \bar{q}_{h}^{1}+\left|\Omega_{2}\right| \bar{q}_{h}^{2}=0$. This yields

$$
-\bar{q}_{h}^{1} \alpha|\Omega|=\left|\Omega_{1}\right||\Omega| \bar{q}_{h}^{1} \bar{p}_{h}^{1}, \quad \text { i.e., } \quad \alpha=-\left|\Omega_{1}\right| \bar{p}_{h}^{1} .
$$

We can cancel by $\bar{q}_{h}^{1}$ because if $\bar{q}_{h}^{1}=0$, then $\bar{q}_{h}=0$. Thus,

$$
|\alpha|=\left|\Omega_{1}\right|^{1 / 2}\left\|\bar{p}_{h}^{1}\right\|_{0, \Omega_{1}} \leq\left|\Omega_{1}\right|^{1 / 2}\left\|p_{h}\right\|_{0, \Omega_{1}} .
$$

For any $E \in \mathcal{E}_{h}$, define $\boldsymbol{R}_{h}(\overline{\boldsymbol{v}}) \in \mathbb{P}_{1}(E)^{2}$ by $\int_{e}\left(\boldsymbol{R}_{h}(\overline{\boldsymbol{v}})-\overline{\boldsymbol{v}}\right)=\mathbf{0}$ on the three edges $e$ of $E$ (i.e., these are the degrees of freedom of the Crouzeix-Raviart element of degree 1). Then $\boldsymbol{R}_{h}(\overline{\boldsymbol{v}}) \in \widetilde{\boldsymbol{X}_{h}}$. Set

$$
\boldsymbol{v}_{h}=\delta \tilde{\boldsymbol{v}}_{h}+\boldsymbol{R}_{h}(\overline{\boldsymbol{v}}) \in \widetilde{\boldsymbol{X}}_{h}, \quad \text { where } \delta>0 \quad \text { is } \quad \text { to be chosen. }
$$

Thus,

$$
b\left(\boldsymbol{v}_{h}, p_{h}\right)=\delta b\left(\tilde{\boldsymbol{v}}_{h}, \tilde{p}_{h}\right)+\delta b\left(\tilde{\boldsymbol{v}}_{h}, \bar{p}_{h}\right)+b\left(\boldsymbol{R}_{h}(\overline{\boldsymbol{v}}), \tilde{p}_{h}\right)+b\left(\boldsymbol{R}_{h}(\overline{\boldsymbol{v}}), \bar{p}_{h}\right) .
$$

First, (4.14) and (4.15) imply

$$
\begin{aligned}
b\left(\tilde{\boldsymbol{v}}_{h}, \bar{p}_{h}\right) & =-\sum_{E \in \mathcal{E}_{h}^{1}} \bar{p}_{h}^{1} \int_{E} \nabla \cdot \tilde{\boldsymbol{v}}_{h}-\sum_{E \in \mathcal{E}_{h}^{2}} \bar{p}_{h}^{2} \int_{E} \nabla \cdot \tilde{\boldsymbol{v}}_{h} \\
& =-\bar{p}_{h}^{1} \sum_{E \in \mathcal{E}_{h}^{1}} \int_{\partial E} \tilde{\boldsymbol{v}}_{h} \cdot \boldsymbol{n}_{E}-\bar{p}_{h}^{2} \sum_{E \in \mathcal{E}_{h}^{2}} \int_{\partial E} \tilde{\boldsymbol{v}}_{h} \cdot \boldsymbol{n}_{E}=0 ;
\end{aligned}
$$


next

$$
\begin{gathered}
b\left(\boldsymbol{R}_{h}(\overline{\boldsymbol{v}}), \bar{p}_{h}\right)=b\left(\boldsymbol{R}_{h}(\overline{\boldsymbol{v}})-\overline{\boldsymbol{v}}, \bar{p}_{h}\right)+b\left(\overline{\boldsymbol{v}}, \bar{p}_{h}\right), \\
b\left(\overline{\boldsymbol{v}}, \bar{p}_{h}\right)=\left\|\bar{p}_{h}\right\|_{0, \Omega}^{2},
\end{gathered}
$$

and it can be easily checked that

$$
b\left(\boldsymbol{R}_{h}(\overline{\boldsymbol{v}})-\overline{\boldsymbol{v}}, \bar{p}_{h}\right)=0 .
$$

Finally,

$$
b\left(\boldsymbol{R}_{h}(\overline{\boldsymbol{v}}), \tilde{p}_{h}\right)=b\left(\boldsymbol{R}_{h}(\overline{\boldsymbol{v}})-\overline{\boldsymbol{v}}, \tilde{p}_{h}\right)-\alpha \sum_{E \in \mathcal{E}_{h}} \int_{E} \tilde{p}_{h} \nabla \cdot \boldsymbol{\rho}
$$

since $\rho$ is continuous. Therefore (4.17) implies

$$
\left|b\left(\overline{\boldsymbol{v}}, \tilde{p}_{h}\right)\right| \leq|\alpha| \sum_{E \in \mathcal{E}_{h}}\left\|\tilde{p}_{h}\right\|_{0, E} \sqrt{2}|\boldsymbol{\rho}|_{1, E} \leq\left|\Omega_{1}\right|^{1 / 2}\left\|\bar{p}_{h}^{1}\right\|_{0, \Omega_{1}} \sqrt{2} K_{1}\left\|\tilde{p}_{h}\right\|_{0, \Omega},
$$

where $K_{1}=|\boldsymbol{\rho}|_{1, \Omega}$. We also denote $K_{2}=|\boldsymbol{\rho}|_{2, \Omega}$. Next

$$
\begin{array}{r}
b\left(\boldsymbol{R}_{h}(\overline{\boldsymbol{v}})-\overline{\boldsymbol{v}}, \tilde{p}_{h}\right)=-\sum_{E \in \mathcal{E}_{h}} \int_{E} \tilde{p}_{h} \nabla \cdot\left(\boldsymbol{R}_{h}(\overline{\boldsymbol{v}})-\overline{\boldsymbol{v}}\right) \\
+\sum_{e \in \Gamma_{h}} \int_{e}\left\{\tilde{p}_{h}\right\}\left[\boldsymbol{R}_{h}(\overline{\boldsymbol{v}})-\overline{\boldsymbol{v}}\right] \cdot \boldsymbol{n}_{e} \\
+\sum_{e \in \gamma_{h}^{1}} \int_{e}\left(\tilde{p}_{h}\right)_{\gamma}\left[\boldsymbol{R}_{h}(\overline{\boldsymbol{v}})-\overline{\boldsymbol{v}}\right] \cdot \boldsymbol{n}_{1}
\end{array}
$$

and it follows from the definition of $\boldsymbol{R}_{h}(\overline{\boldsymbol{v}})$ and (4.17) that

$$
\begin{aligned}
\left|\int_{E} \tilde{p}_{h} \nabla \cdot\left(\boldsymbol{R}_{h}(\overline{\boldsymbol{v}})-\overline{\boldsymbol{v}}\right)\right| & \leq\left\|\tilde{p}_{h}\right\|_{0, E} \sqrt{2} \hat{C} h_{E}|\overline{\boldsymbol{v}}|_{2, E} \\
& \leq \sqrt{2} \hat{C} h_{E}\left\|\tilde{p}_{h}\right\|_{0, E}\left|\Omega_{1}\right|^{1 / 2}\left\|\bar{p}_{h}^{1}\right\|_{0, \Omega_{1}}|\boldsymbol{\rho}|_{2, E} .
\end{aligned}
$$

Therefore

$$
\sum_{E \in \mathcal{E}_{h}}\left|\int_{E} \tilde{p}_{h} \nabla \cdot\left(\boldsymbol{R}_{h}(\overline{\boldsymbol{v}})-\overline{\boldsymbol{v}}\right)\right| \leq \sqrt{2} \hat{C} K_{2} h\left|\Omega_{1}\right|^{1 / 2}\left\|\tilde{p}_{h}\right\|_{0, \Omega}\left\|\bar{p}_{h}^{1}\right\|_{0, \Omega_{1}}
$$

In addition, applying (4.5) to this choice of $\boldsymbol{R}_{h}$ and adding (4.20), we obtain

$$
\left|b\left(\boldsymbol{R}_{h}(\overline{\boldsymbol{v}})-\overline{\boldsymbol{v}}, \tilde{p}_{h}\right)\right| \leq \hat{C} h K_{2}\left|\Omega_{1}\right|^{1 / 2}\left\|\tilde{p}_{h}\right\|_{0, \Omega}\left\|\bar{p}_{h}^{1}\right\|_{0, \Omega_{1}},
$$

with a constant $\hat{C}$ that does not depend on $h$. Collecting the relations (4.16), (4.18), (4.19) and (4.21), we derive

$$
\begin{aligned}
b\left(\boldsymbol{v}_{h}, p_{h}\right) \geq & \delta\left\|\tilde{p}_{h}\right\|_{0, \Omega}^{2}+\left\|\bar{p}_{h}\right\|_{0, \Omega}^{2}-\sqrt{2} K_{1}\left|\Omega_{1}\right|^{1 / 2}\left\|\bar{p}_{h}^{1}\right\|_{0, \Omega_{1}}\left\|\tilde{p}_{h}\right\|_{0, \Omega} \\
& -\hat{C} h K_{2}\left|\Omega_{1}\right|^{1 / 2}\left\|\bar{p}_{h}^{1}\right\|_{0, \Omega_{1}}\left\|\tilde{p}_{h}\right\|_{0, \Omega} \\
\geq & \delta\left\|\tilde{p}_{h}\right\|_{0, \Omega}^{2}+\left\|\bar{p}_{h}\right\|_{0, \Omega}^{2}-\sqrt{2} K_{1}\left|\Omega_{1}\right|^{1 / 2} \frac{1}{2}\left(\lambda\left\|\bar{p}_{h}^{1}\right\|_{0, \Omega_{1}}^{2}+\frac{1}{\lambda}\left\|\tilde{p}_{h}\right\|_{0, \Omega}^{2}\right) \\
& -\hat{C} h K_{2}\left|\Omega_{1}\right|^{1 / 2} \frac{1}{2}\left(\lambda^{\prime}\left\|\bar{p}_{h}^{1}\right\|_{0, \Omega_{1}}^{2}+\frac{1}{\lambda^{\prime}}\left\|\tilde{p}_{h}\right\|_{0, \Omega}^{2}\right), \quad \lambda>0, \quad \lambda^{\prime}>0, \\
\geq & \left\|\tilde{p}_{h}\right\|_{0, \Omega}^{2}\left(\delta-\frac{\sqrt{2}}{2} K_{1}\left|\Omega_{1}\right|^{1 / 2} \frac{1}{\lambda}-\hat{C} h \frac{K_{2}}{2}\left|\Omega_{1}\right|^{1 / 2} \frac{1}{\lambda^{\prime}}\right) \\
& +\left\|\bar{p}_{h}\right\|_{0, \Omega}^{2}\left(1-\frac{\sqrt{2}}{2} K_{1}|\Omega|^{1 / 2} \lambda-\hat{C} \frac{h}{2} K_{2}\left|\Omega_{1}\right|^{1 / 2} \lambda^{\prime}\right) .
\end{aligned}
$$


Choose for instance $\lambda$ and $\lambda^{\prime}$ so that

$$
\frac{\sqrt{2}}{2} K_{1}\left|\Omega_{1}\right|^{1 / 2} \lambda+\hat{C} \frac{h}{2} K_{2}\left|\Omega_{1}\right|^{1 / 2} \lambda^{\prime}=\frac{1}{2}
$$

for example

$$
\lambda=\frac{1}{2 \sqrt{2}} \frac{1}{K_{1}\left|\Omega_{1}\right|^{1 / 2}}, \quad \lambda^{\prime}=\frac{1}{2 \hat{C} h K_{2}\left|\Omega_{1}\right|^{1 / 2}} .
$$

Then, the coefficient of $\left\|\tilde{p}_{h}\right\|_{0, \Omega}^{2}$ is $\delta-2 K_{1}^{2}\left|\Omega_{1}\right|-\hat{C}^{2} h^{2} K_{2}^{2}\left|\Omega_{1}\right|$. Choose for instance $\delta=2 K_{1}^{2}\left|\Omega_{1}\right|+\hat{C}^{2} h^{2} K_{2}^{2}\left|\Omega_{1}\right|+\frac{1}{2}$. Then,

$$
b\left(\boldsymbol{v}_{h}, p_{h}\right) \geq \frac{1}{2}\left\|\tilde{p}_{h}\right\|_{0}^{2}+\frac{1}{2}\left\|\bar{p}_{h}\right\|_{0}^{2}=\frac{1}{2}\left\|p_{h}\right\|_{0}^{2} .
$$

This establishes (4.9) with $\beta_{1}^{*}=\frac{1}{2}$. There remains to bound $\llbracket \boldsymbol{v}_{h} \rrbracket$. We first bound $\left(\sum_{E \in \mathcal{E}_{h}}\left|\boldsymbol{v}_{h}\right|_{1, E}^{2}\right)^{1 / 2}:$

$$
\left|\boldsymbol{v}_{h}\right|_{1, E} \leq\left(2 K_{1}^{2}\left|\Omega_{1}\right|+\hat{C}^{2} h^{2} K_{2}^{2}\left|\Omega_{1}\right|+\frac{1}{2}\right)\left|\tilde{\boldsymbol{v}}_{h}\right|_{1, E}+\left|\boldsymbol{R}_{h}(\overline{\boldsymbol{v}})\right|_{1, E} .
$$

But (3.15) with $s=1$ and $k=1$ implies

$$
\left|\boldsymbol{R}_{h}(\overline{\boldsymbol{v}})\right|_{1, E} \leq C_{2}|\overline{\boldsymbol{v}}|_{1, E} \leq C_{2}\left|\Omega_{1}\right|^{1 / 2}\left\|\bar{p}_{h}^{1}\right\|_{0, \Omega_{1}}|\boldsymbol{\rho}|_{1, E} .
$$

Then,

$$
\left\|\nabla \boldsymbol{v}_{h}\right\|_{0} \leq\left(\frac{C_{3}^{2}}{\beta^{2}}+C_{4}^{2}\right)^{1 / 2}\left\|\tilde{p}_{h}\right\|_{0}
$$

where

$$
C_{3}=\sqrt{2} C_{1}\left(\frac{1}{2}+\left|\Omega_{1}\right|\left(2 K_{1}^{2}+h^{2} \hat{C}^{2} K_{2}^{2}\right)\right), \quad C_{4}=\sqrt{2} C_{2}\left|\Omega_{1}\right|^{1 / 2} K_{1} .
$$

We now bound $J_{0}\left(\boldsymbol{v}_{h}, \boldsymbol{v}_{h}\right)$. Since $J_{0}$ is a seminorm and $\boldsymbol{v}_{h}$ is the sum of $\boldsymbol{R}_{h}(\overline{\boldsymbol{v}})$ and $\delta \tilde{\boldsymbol{v}}_{h}$, it suffices to bound $J_{0}\left(\tilde{\boldsymbol{v}}_{h}, \tilde{\boldsymbol{v}}_{h}\right)$ and $J_{0}\left(\boldsymbol{R}_{h}(\overline{\boldsymbol{v}}), \boldsymbol{R}_{h}(\overline{\boldsymbol{v}})\right)$. As $\tilde{\boldsymbol{v}}_{i} \in H_{0}^{1}\left(\Omega_{i}\right)^{2}$, we can write

$$
J_{0}\left(\tilde{\boldsymbol{v}}_{h}, \tilde{\boldsymbol{v}}_{h}\right) \leq 2 \sum_{i=1}^{2} \sum_{e \in \Gamma_{h}^{i} \cup \gamma_{h}^{i}} \frac{\sigma_{e}}{|e|}\left\|\tilde{\boldsymbol{v}}^{i}-\boldsymbol{R}_{h}^{i}\left(\tilde{\boldsymbol{v}}^{i}\right)\right\|_{0, e}^{2} .
$$

Lemma [3.9 and (4.4) together with (3.15) with $s=1$ and (4.13) imply that

$$
\delta J_{0}\left(\tilde{\boldsymbol{v}}_{h}, \tilde{\boldsymbol{v}}_{h}\right)^{1 / 2} \leq \frac{C_{5}}{\beta}\left\|\tilde{p}_{h}\right\|_{0, \Omega},
$$

with a constant $C_{5}$ independent of $h$ and $\beta$. The argument for bounding $J_{0}\left(\boldsymbol{R}_{h}(\overline{\boldsymbol{v}}), \boldsymbol{R}_{h}(\overline{\boldsymbol{v}})\right)$ is a combination of Lemmas 3.9 and 4.2. Let $e \in \gamma_{h}^{1}$; we have

$$
\sum_{e \in \gamma_{h}^{1}} \frac{1}{|e|}\left\|\left[\boldsymbol{R}_{h}(\overline{\boldsymbol{v}})\right]\right\|_{0, e}^{2}=\sum_{e \in \gamma_{h}^{1}} \frac{1}{|e|}\left\|\left[\boldsymbol{R}_{h}(\overline{\boldsymbol{v}})-\overline{\boldsymbol{v}}\right]\right\|_{0, e}^{2}
$$

Then proceeding as in Lemma 4.2, we write

$$
\begin{aligned}
\frac{\sigma_{e}}{|e|} \| & {\left[\boldsymbol{R}_{h}(\overline{\boldsymbol{v}})\right]\left\|_{0, e}^{2}=\frac{\sigma_{e}}{|e|} \sum_{j} \int_{e e_{j}}\right\|\left(\boldsymbol{R}_{h}(\overline{\boldsymbol{v}})-\overline{\boldsymbol{v}}\right)\left|\Omega_{\Omega_{1}}-\left(\boldsymbol{R}_{h}(\overline{\boldsymbol{v}})-\overline{\boldsymbol{v}}\right)\right|_{\Omega_{2}} \|^{2} } \\
& \leq \frac{2 \sigma_{e}}{|e|}\left\|\left.\left(\boldsymbol{R}_{h}(\overline{\boldsymbol{v}})-\overline{\boldsymbol{v}}\right)\right|_{\Omega_{1}}\right\|_{0, e}^{2}+2 \sigma_{m} \sum_{j} \frac{\left|e_{j}\right|}{|e|} \frac{1}{\left|e_{j}\right|}\left\|\left.\left(\boldsymbol{R}_{h}(\overline{\boldsymbol{v}})-\overline{\boldsymbol{v}}\right)\right|_{\Omega_{2}}\right\|_{0, e_{j}}^{2} \\
& \leq \frac{2 \sigma_{e}}{|e|}\left\|\left.\left(\boldsymbol{R}_{h}(\overline{\boldsymbol{v}})-\overline{\boldsymbol{v}}\right)\right|_{\Omega_{1}}\right\|_{0, e}^{2}+2 L_{2} \sigma_{m} \sum_{j} \frac{1}{\left|e_{j}\right|}\left\|\left.\left(\boldsymbol{R}_{h}(\overline{\boldsymbol{v}})-\overline{\boldsymbol{v}}\right)\right|_{\Omega_{2}}\right\|_{0, e_{j}}^{2},
\end{aligned}
$$


where $j$ runs over all segments $e_{j}$ of $\gamma_{h}^{2}$ that intersect $e$. Therefore

$$
\sum_{e \in \gamma_{h}^{1}} \frac{1}{|e|}\left\|\left[\boldsymbol{R}_{h}(\overline{\boldsymbol{v}})\right]\right\|_{0, e}^{2} \leq \hat{C}\left\|\nabla\left(\boldsymbol{R}_{h}(\overline{\boldsymbol{v}})-\overline{\boldsymbol{v}}\right)\right\|_{0, D_{1} \cup D_{2}}^{2},
$$

with a constant $\hat{C}$ that depends on $L_{2}$, but not on $h$. If $e \in \Gamma_{h}^{i}$, we use the argument of Lemma 3.9. Then, (3.15) with $s=1, k=1$ and (4.17) imply

$$
J_{0}\left(\boldsymbol{R}_{h}(\overline{\boldsymbol{v}}), \boldsymbol{R}_{h}(\overline{\boldsymbol{v}})\right)^{1 / 2} \leq C_{6}\left\|\bar{p}_{h}^{1}\right\|_{0, \Omega_{1}}
$$

where $C_{6}=\hat{C} K_{1}\left|\Omega_{1}\right|^{1 / 2}$. Hence

$$
J_{0}\left(\boldsymbol{v}_{h}, \boldsymbol{v}_{h}\right)^{1 / 2} \leq\left(\frac{C_{5}^{2}}{\beta^{2}}+C_{6}^{2}\right)^{1 / 2}\left\|p_{h}\right\|_{0, \Omega} .
$$

This proves 4.10 with $\beta_{2}^{*}=\left(\frac{C_{3}^{2}}{\beta^{2}}+\frac{C_{5}^{2}}{\beta^{2}}+C_{4}^{2}+C_{6}^{2}\right)^{1 / 2}$.

Remark 4.6. Theorem 4.5 establishes the inf-sup condition for two subdomains. It can be extended by induction to a fixed number of subdomains.

Remark 4.7. An immediate consequence of the inf-sup condition (4.8) is [12]: for any $p_{h}$ in $M_{h}$, there exists a unique $\boldsymbol{v}_{h}$ in $\widetilde{\boldsymbol{X}_{h}}$ such that

$$
\begin{gathered}
\forall \boldsymbol{w}_{h} \in \boldsymbol{V}_{h}, \quad \sum_{E \in \mathcal{E}_{h}} \int_{E} \nabla \boldsymbol{v}_{h}: \nabla \boldsymbol{w}_{h}+J_{0}\left(\boldsymbol{v}_{h}, \boldsymbol{w}_{h}\right)=0, \\
b\left(\boldsymbol{v}_{h}, p_{h}\right)=-\left\|p_{h}\right\|_{0}^{2}, \quad \llbracket \mid \boldsymbol{v}_{h}\left\|\leq \frac{1}{\beta^{*}}\right\| p_{h} \|_{0} .
\end{gathered}
$$

The next corollary shows that the inf-sup condition allows one to construct a good approximation operator.

Corollary 4.8. Under the assumptions of Theorem 4.5. there exists an approximation operator $\boldsymbol{P}_{h} \in \mathcal{L}\left(H_{0}^{1}(\Omega)^{2} ; \widetilde{\boldsymbol{X}_{h}}\right)$ such that for any $s \in[1, k+1]$ :

$$
\begin{gathered}
\forall \boldsymbol{v} \in H_{0}^{1}(\Omega)^{2}, \quad \forall q_{h} \in M_{h}, \quad b\left(\boldsymbol{P}_{h}(\boldsymbol{v})-\boldsymbol{v}, q_{h}\right)=0, \\
\forall \boldsymbol{v} \in\left(H^{s}(\Omega) \cap H_{0}^{1}(\Omega)\right)^{2}, \quad \llbracket \boldsymbol{P}_{h}(\boldsymbol{v})-\boldsymbol{v} \rrbracket \leq C h^{s-1}|\boldsymbol{v}|_{s, \Omega}, \\
\forall \boldsymbol{v} \in H_{0}^{1}(\Omega)^{2}, \quad \forall e \in \Gamma_{h}, \quad \forall \boldsymbol{q} \in \mathbb{P}_{k-1}(e)^{2}, \quad \int_{e}\left[\boldsymbol{P}_{h}(\boldsymbol{v})-\boldsymbol{v}\right] \cdot \boldsymbol{q}=0 .
\end{gathered}
$$

Proof. For $k=1,2$ or 3 , let $\boldsymbol{v} \in H_{0}^{1}(\Omega)^{2}$ and let $\boldsymbol{R}_{h}(\boldsymbol{v})$ be the interpolant of $\boldsymbol{v}$ in $\boldsymbol{X}_{h}$ satisfying (3.12)-(3.15), defined separately in $\Omega_{1}$ and $\Omega_{2}$. Then, we define

$$
\boldsymbol{P}_{h}(\boldsymbol{v})=\boldsymbol{R}_{h}(\boldsymbol{v})+\boldsymbol{c}_{h},
$$

where $\boldsymbol{c}_{h} \in \widetilde{\boldsymbol{X}_{h}}$ satisfies

$$
\forall q_{h} \in M_{h}, \quad b\left(\boldsymbol{c}_{h}, q_{h}\right)=b\left(\boldsymbol{v}-\boldsymbol{R}_{h}(\boldsymbol{v}), q_{h}\right) .
$$

Theorem 4.5 implies that $\boldsymbol{c}_{h} \in \widetilde{\boldsymbol{X}_{h}}$ exists and

$$
\llbracket \boldsymbol{c}_{h} \rrbracket \leq \frac{1}{\beta^{*}} \sup _{q_{h} \in M_{h}} \frac{b\left(\boldsymbol{v}-\boldsymbol{R}_{h}(\boldsymbol{v}), q_{h}\right)}{\left\|q_{h}\right\|_{0}} .
$$


Furthermore,

$$
\begin{aligned}
b\left(\boldsymbol{v}-\boldsymbol{R}_{h}(\boldsymbol{v}), q_{h}\right) \leq \sqrt{2}\left\|q_{h}\right\|_{0, \Omega}\left\|\nabla\left(\boldsymbol{v}-\boldsymbol{R}_{h}(\boldsymbol{v})\right)\right\|_{0} & +\left|\sum_{e \in \Gamma_{h}} \int_{e}\left\{q_{h}\right\}\left[\boldsymbol{v}-\boldsymbol{R}_{h}(\boldsymbol{v})\right] \cdot \boldsymbol{n}_{e}\right| \\
& +\left|\sum_{e \in \gamma_{h}^{1}} \int_{e}\left(q_{h}\right)_{\gamma}\left[\boldsymbol{v}-\boldsymbol{R}_{h}(\boldsymbol{v})\right] \cdot \boldsymbol{n}_{1}\right| .
\end{aligned}
$$

With Lemma 4.2 and Remark 4.4 this becomes

$$
\left|b\left(\boldsymbol{v}-\boldsymbol{R}_{h}(\boldsymbol{v}), q_{h}\right)\right| \leq C\left\|q_{h}\right\|_{0}\left\|\nabla\left(\boldsymbol{v}-\boldsymbol{R}_{h}(\boldsymbol{v})\right)\right\|_{0} .
$$

Then (4.23) follows from the argument used in bounding $J_{0}\left(\boldsymbol{R}_{h}(\overline{\boldsymbol{v}}), \boldsymbol{R}_{h}(\overline{\boldsymbol{v}})\right)$ in Lemma 3.9 and the approximation properties of $\boldsymbol{R}_{h}$, and (4.24) follows from (3.13) and the fact that $\boldsymbol{c}_{h} \in \widetilde{\boldsymbol{X}_{h}}$.

\section{ERror estimates}

In this section, we derive optimal a priori error estimates in the energy norm of the error for the velocity field and the $L^{2}$ norm of the error for the pressure. A third estimate gives an optimal convergence rate for the $L^{2}$ norm of the error in the velocity field for the symmetric formulation $\left(\epsilon^{*}=-1\right)$ and a nonoptimal convergence rate for the nonsymmetric formulation.

Theorem 5.1. Let $k=1,2$ or 3 be the degree of the polynomials in the definition of $\boldsymbol{X}_{h}$ and assume that the solution $(\boldsymbol{u}, p)$ of problem (2.1) (2.3) belongs to $H^{k+1}(\Omega)^{2} \times H^{k}(\Omega)$. Then, if the triangulation satisfies (3.1) and Hypotheses $\mathrm{H} 1$ and $\mathrm{H} 2$, and if $\mathrm{H} 3$ holds, the solution $(\boldsymbol{U}, P)$ of (3.8), (3.9) satisfies the error estimate

$$
\llbracket \boldsymbol{u}-\boldsymbol{U} \rrbracket \leq C h^{k}\left(|\boldsymbol{u}|_{k+1}+\frac{1}{\mu}|p|_{k}\right),
$$

where $C$ is independent of $h$ and $\mu$.

Proof. Let $\boldsymbol{U}_{I}=\boldsymbol{P}_{h}(\boldsymbol{u})$ and $P_{I}=r_{h}(p)$. Denote $\boldsymbol{\chi}=\boldsymbol{U}-\boldsymbol{U}_{I}, \xi=P-P_{I}$. The errors $\chi$ and $\xi$ satisfy the equations

$$
\begin{aligned}
\mu\left(a(\boldsymbol{\chi}, \boldsymbol{v})+J_{0}(\boldsymbol{\chi}, \boldsymbol{v})\right)+b(\boldsymbol{v}, \xi)= & \mu\left(a\left(\boldsymbol{u}-\boldsymbol{U}_{I}, \boldsymbol{v}\right)+J_{0}\left(\boldsymbol{u}-\boldsymbol{U}_{I}, \boldsymbol{v}\right)\right) \\
& +b\left(\boldsymbol{v}, p-P_{I}\right), \quad \forall \boldsymbol{v} \in \boldsymbol{X}_{h}, \\
b(\boldsymbol{\chi}, q)= & b\left(\boldsymbol{u}-\boldsymbol{U}_{I}, q\right), \quad \forall q \in M_{h} .
\end{aligned}
$$

Set $\boldsymbol{v}=\chi$ and $q=\xi$. Then dividing by $\mu$, the two error equations become by virtue of (4.22) and (4.7):

$$
K \llbracket \boldsymbol{\chi} \rrbracket^{2} \leq a(\boldsymbol{\chi}, \boldsymbol{\chi})+J_{0}(\boldsymbol{\chi}, \boldsymbol{\chi})=a\left(\boldsymbol{u}-\boldsymbol{U}_{I}, \boldsymbol{\chi}\right)+J_{0}\left(\boldsymbol{u}-\boldsymbol{U}_{I}, \boldsymbol{\chi}\right)+\frac{1}{\mu} b\left(\boldsymbol{\chi}, p-P_{I}\right) .
$$

Then, we only need to bound the three terms $a\left(\boldsymbol{u}-\boldsymbol{U}_{I}, \boldsymbol{\chi}\right), J_{0}\left(\boldsymbol{u}-\boldsymbol{U}_{I}, \boldsymbol{\chi}\right)$ and $\frac{1}{\mu} b\left(\chi, p-P_{I}\right)$. By definition,

$$
\begin{aligned}
a\left(\boldsymbol{u}-\boldsymbol{U}_{I}, \boldsymbol{\chi}\right)= & \sum_{E \in \mathcal{E}_{h}} \int_{E} \nabla\left(\boldsymbol{u}-\boldsymbol{U}_{I}\right): \nabla \boldsymbol{\chi}-\sum_{e \in \Gamma_{h} \cup \gamma_{h}^{1}} \int_{e}\left\{\nabla\left(\boldsymbol{u}-\boldsymbol{U}_{I}\right)\right\} \boldsymbol{n}_{e} \cdot[\boldsymbol{\chi}] \\
& +\epsilon^{*} \sum_{e \in \Gamma_{h} \cup \gamma_{h}^{1}} \int_{e}\{\nabla \boldsymbol{\chi}\} \boldsymbol{n}_{e} \cdot\left[\boldsymbol{u}-\boldsymbol{U}_{I}\right] \\
= & T_{1}+T_{2}+T_{3} .
\end{aligned}
$$


Using Cauchy-Schwarz and the approximation result (4.23), we have

$$
\begin{aligned}
T_{1} & \leq \sum_{E \in \mathcal{E}_{h}}\left\|\nabla\left(\boldsymbol{u}-\boldsymbol{U}_{I}\right)\right\|_{0, E}\|\nabla \boldsymbol{\chi}\|_{0, E} \leq \frac{K}{8}\|\nabla \boldsymbol{\chi}\|_{0}^{2}+C\left\|\nabla\left(\boldsymbol{u}-\boldsymbol{U}_{I}\right)\right\|_{0}^{2} \\
& \leq \frac{K}{8}\|\nabla \boldsymbol{\chi}\|_{0}^{2}+C h^{2 k}|\boldsymbol{u}|_{k+1}^{2} .
\end{aligned}
$$

Let $L_{h}(\boldsymbol{u})$ denote the standard Lagrange interpolant of degree $k$ defined separately in $\Omega_{1}$ and $\Omega_{2}$ and let us insert it in the second integral term:

$$
\int_{e}\left\{\nabla\left(\boldsymbol{u}-\boldsymbol{U}_{I}\right)\right\} \boldsymbol{n}_{e} \cdot[\boldsymbol{\chi}]=\int_{e}\left\{\nabla\left(\boldsymbol{u}-L_{h}(\boldsymbol{u})\right)\right\} \boldsymbol{n}_{e} \cdot[\boldsymbol{\chi}]+\int_{e}\left\{\nabla\left(L_{h}(\boldsymbol{u})-\boldsymbol{U}_{I}\right)\right\} \boldsymbol{n}_{e} \cdot[\boldsymbol{\chi}] .
$$

Let $e$ be a segment of $\gamma_{h}^{1}$. Expanding the first integral and applying the argument of Lemma 4.2, we obtain with the same notation

$$
\begin{gathered}
\left|\int_{e}\left\{\nabla\left(\boldsymbol{u}-L_{h}(\boldsymbol{u})\right)\right\} \boldsymbol{n}_{e} \cdot[\boldsymbol{\chi}]\right| \leq \frac{1}{2} \hat{C}\left(1+L_{2}\right)^{1 / 2}\left(\left|\boldsymbol{u}-L_{h}(\boldsymbol{u})\right|_{1, E}^{2}\right. \\
\left.+h_{E}^{2}\left|\boldsymbol{u}-L_{h}(\boldsymbol{u})\right|_{2, E}^{2}+\sum_{j}\left(\left|\boldsymbol{u}-L_{h}(\boldsymbol{u})\right|_{1, E_{j}}^{2}+h_{E_{j}}^{2}\left|\boldsymbol{u}-L_{h}(\boldsymbol{u})\right|_{2, E_{j}}^{2}\right)\right)^{1 / 2} \frac{\sigma_{e}^{1 / 2}}{|e|^{1 / 2}}\|[\boldsymbol{\chi}]\|_{0, e} .
\end{gathered}
$$

Similarly,

$$
\begin{gathered}
\left|\int_{e}\left\{\nabla\left(L_{h}(\boldsymbol{u})-\boldsymbol{U}_{I}\right)\right\} \boldsymbol{n}_{e} \cdot[\boldsymbol{\chi}]\right| \leq \frac{1}{2} \hat{C}\left(1+L_{2}\right)^{1 / 2}\left(\left|L_{h}(\boldsymbol{u})-\boldsymbol{U}_{I}\right|_{1, E}^{2}\right. \\
\left.+\sum_{j}\left|L_{h}(\boldsymbol{u})-\boldsymbol{U}_{I}\right|_{1, E_{j}}^{2}\right)^{1 / 2} \frac{\sigma_{e}^{1 / 2}}{|e|^{1 / 2}}\|[\boldsymbol{\chi}]\|_{0, e} .
\end{gathered}
$$

Therefore,

$$
\begin{gathered}
\left|\sum_{e \in \Gamma_{h} \cup \gamma_{h}^{1}} \int_{e}\left\{\nabla\left(\boldsymbol{u}-\boldsymbol{U}_{I}\right)\right\} \boldsymbol{n}_{e} \cdot[\boldsymbol{\chi}]\right| \leq \hat{C}\left(\left\|\nabla\left(\boldsymbol{u}-L_{h}(\boldsymbol{u})\right)\right\|_{0}^{2}+\left\|\nabla\left(L_{h}(\boldsymbol{u})-\boldsymbol{U}_{I}\right)\right\|_{0}^{2}\right. \\
\left.+\sum_{E \in \mathcal{E}_{h}} h_{E}^{2}\left|\boldsymbol{u}-L_{h}(\boldsymbol{u})\right|_{2, E}^{2}\right)^{1 / 2} J_{0}(\boldsymbol{\chi}, \boldsymbol{\chi})^{1 / 2} .
\end{gathered}
$$

Hence the estimate (4.23) and the standard approximation properties of $L_{h}$ yield

$$
\left|T_{2}\right| \leq \frac{K}{8} J_{0}(\boldsymbol{\chi}, \boldsymbol{\chi})+C h^{2 k}|\boldsymbol{u}|_{k+1}^{2}
$$

The third term has the same structure as the one studied in Lemma 4.2 because the degree of $\{\nabla \boldsymbol{\chi}\} \boldsymbol{n}_{e}$ is $k-1$; thus it satisfies the bound

$$
\left|T_{3}\right| \leq C\|\nabla \boldsymbol{\chi}\|_{0, D^{12}}\left\|\nabla\left(\boldsymbol{u}-\boldsymbol{U}_{I}\right)\right\|_{0, D^{12}} \leq \frac{K}{8}\|\nabla \boldsymbol{\chi}\|_{0}^{2}+C h^{2 k}|\boldsymbol{u}|_{k+1}^{2} .
$$

Using Cauchy-Schwarz inequality, the jump term is bounded by virtue of (4.23):

$$
J_{0}\left(\boldsymbol{u}-\boldsymbol{U}_{I}, \boldsymbol{\chi}\right) \leq \frac{K}{8} J_{0}(\boldsymbol{\chi}, \boldsymbol{\chi})+C J_{0}\left(\boldsymbol{u}-\boldsymbol{U}_{I}, \boldsymbol{u}-\boldsymbol{U}_{I}\right) \leq \frac{K}{8} J_{0}(\boldsymbol{\chi}, \boldsymbol{\chi})+C h^{2 k}|\boldsymbol{u}|_{k+1}^{2} .
$$

Finally, the term involving the pressure reduces to

$$
\frac{1}{\mu} b\left(\boldsymbol{\chi}, p-P_{I}\right)=\frac{1}{\mu} \sum_{e \in \Gamma_{h}} \int_{e}\left\{p-P_{I}\right\}[\chi] \cdot \boldsymbol{n}_{e}+\frac{1}{\mu} \sum_{e \in \gamma_{h}^{1}} \int_{e}\left(p-P_{I}\right)_{\gamma}[\boldsymbol{\chi}] \cdot \boldsymbol{n}_{e},
$$


owing to (3.10) and the fact that each component of $\nabla \cdot \chi \in \mathbb{P}_{k-1}(E)$. Its righthand side is similar (but simpler than) $T_{2}$; the first term is bounded by

$$
\begin{aligned}
\frac{1}{\mu}\left|\sum_{e \in \Gamma_{h}} \int_{e}\left\{p-P_{I}\right\}[\chi] \cdot \boldsymbol{n}_{e}\right| & \leq \frac{\hat{C}}{\mu}\left(\left\|p-P_{I}\right\|_{0}^{2}+\sum_{E \in \mathcal{E}_{h}} h_{E}^{2}\left|p-P_{I}\right|_{1, E}^{2}\right)^{1 / 2} J_{0}(\boldsymbol{\chi}, \boldsymbol{\chi})^{1 / 2} \\
& \leq \frac{K}{8} J_{0}(\boldsymbol{\chi}, \boldsymbol{\chi})+\frac{C}{\mu^{2}} h^{2 k}|p|_{k}^{2} .
\end{aligned}
$$

Similarly

$$
\frac{1}{\mu}\left|\sum_{e \in \gamma_{h}^{1}} \int_{e}\left(p-P_{I}\right)_{\gamma}[\boldsymbol{\chi}] \cdot \boldsymbol{n}_{e}\right| \leq \frac{K}{8} J_{0}(\boldsymbol{\chi}, \boldsymbol{\chi})+\frac{C}{\mu^{2}} h^{2 k}|p|_{k}^{2} .
$$

Combining all the bounds above, we obtain

$$
K \llbracket \boldsymbol{\chi}\left\|^{2} \leq \frac{K}{2} J_{0}(\boldsymbol{\chi}, \boldsymbol{\chi})+\frac{K}{4}\right\| \nabla \boldsymbol{\chi} \|_{0}^{2}+C h^{2 k}\left(|\boldsymbol{u}|_{k+1}^{2}+\frac{1}{\mu^{2}}|p|_{k}^{2}\right)
$$

therefore

$$
\llbracket \chi \rrbracket^{2} \leq C h^{2 k}\left(|\boldsymbol{u}|_{k+1}^{2}+\frac{1}{\mu^{2}}|p|_{k}^{2}\right)
$$

Then (5.1) follows from the triangle inequality and (4.23).

We now derive an estimate for the pressure.

Theorem 5.2. Under the assumptions and notation of Theorem 5.1, we have

$$
\|p-P\|_{0} \leq C h^{k}\left(\mu|\boldsymbol{u}|_{k+1}+|p|_{k}\right)
$$

with a constant $C$ independent of $h$ and $\mu$.

Proof. We can write the error equation as follows:

$$
\forall \boldsymbol{v} \in \boldsymbol{X}_{h}, \quad a(\boldsymbol{U}-\boldsymbol{u}, \boldsymbol{v})+J_{0}(\boldsymbol{U}-\boldsymbol{u}, \boldsymbol{v})+\frac{1}{\mu} b\left(\boldsymbol{v}, P-P_{I}\right)=\frac{1}{\mu} b\left(\boldsymbol{v}, p-P_{I}\right) .
$$

By virtue of Remark 4.7, there exists $\boldsymbol{v}_{h} \in \widetilde{\boldsymbol{X}_{h}}$ such that

$$
b\left(\boldsymbol{v}_{h}, P-P_{I}\right)=-\left\|P-P_{I}\right\|_{0}^{2}, \quad \llbracket\left\|\boldsymbol{v}_{h}\right\| \leq \frac{1}{\beta^{*}}\left\|P-P_{I}\right\|_{0},
$$

and in particular

$$
\sum_{E \in \mathcal{E}_{h}} \int_{E} \nabla\left(\boldsymbol{U}-\boldsymbol{U}_{I}\right): \nabla \boldsymbol{v}_{h}+J_{0}\left(\boldsymbol{U}-\boldsymbol{U}_{I}, \boldsymbol{v}_{h}\right)=0
$$

where $\boldsymbol{U}_{I}=\boldsymbol{P}_{h}(\boldsymbol{u})$. Therefore, (5.4) with the choice $\boldsymbol{v}=\boldsymbol{v}_{h}$ becomes

$$
\begin{aligned}
\frac{1}{\mu}\left\|P-P_{I}\right\|_{0}^{2}= & a\left(\boldsymbol{U}-\boldsymbol{u}, \boldsymbol{v}_{h}\right)+J_{0}\left(\boldsymbol{U}-\boldsymbol{u}, \boldsymbol{v}_{h}\right)-\frac{1}{\mu} b\left(\boldsymbol{v}_{h}, p-P_{I}\right) \\
= & \sum_{E \in \mathcal{E}_{h}} \int_{E} \nabla\left(\boldsymbol{U}_{I}-\boldsymbol{u}\right): \nabla \boldsymbol{v}_{h}+J_{0}\left(\boldsymbol{U}_{I}-\boldsymbol{u}, \boldsymbol{v}_{h}\right)-\frac{1}{\mu} b\left(\boldsymbol{v}_{h}, p-P_{I}\right) \\
& -\sum_{e \in \Gamma \cup \gamma_{h}^{1}} \int_{e}\{\nabla(\boldsymbol{U}-\boldsymbol{u})\} \boldsymbol{n}_{e} \cdot\left[\boldsymbol{v}_{h}\right]+\epsilon^{*} \sum_{e \in \Gamma \cup \gamma_{h}^{1}} \int_{e}\left\{\nabla \boldsymbol{v}_{h}\right\} \boldsymbol{n}_{e} \cdot[\boldsymbol{U}-\boldsymbol{u}] \\
= & Q_{1}+Q_{2}+\cdots+Q_{5} .
\end{aligned}
$$


The estimates for $Q_{1}, Q_{2}$ and $Q_{3}$ are straightforward. The bound for $Q_{5}$ is similar, but simpler than that for $T_{2}$ :

$$
\left|Q_{5}\right| \leq C J_{0}(\boldsymbol{U}-\boldsymbol{u}, \boldsymbol{U}-\boldsymbol{u})^{1 / 2}\left\|\nabla \boldsymbol{v}_{h}\right\|_{0} \leq \frac{C}{\beta^{*}} J_{0}(\boldsymbol{U}-\boldsymbol{u}, \boldsymbol{U}-\boldsymbol{u})^{1 / 2}\left\|P-P_{I}\right\|_{0} .
$$

We then conclude by using (5.1):

$$
\left|Q_{5}\right| \leq C h^{k}\left(|\boldsymbol{u}|_{k+1}+\frac{1}{\mu}|p|_{k}\right)\left\|P-P_{I}\right\|_{0} .
$$

It remains to bound $Q_{4}$; we write

$$
\int_{e}\{\nabla(\boldsymbol{U}-\boldsymbol{u})\} \boldsymbol{n}_{e} \cdot\left[\boldsymbol{v}_{h}\right]=\int_{e}\left\{\nabla\left(\boldsymbol{U}-\boldsymbol{U}_{I}\right)\right\} \boldsymbol{n}_{e} \cdot\left[\boldsymbol{v}_{h}\right]+\int_{e}\left\{\nabla\left(\boldsymbol{U}_{I}-\boldsymbol{u}\right)\right\} \boldsymbol{n}_{e} \cdot\left[\boldsymbol{v}_{h}\right] .
$$

The second term is bounded as $T_{2}$ in Theorem 5.1

$$
\left|\sum_{e \in \Gamma \cup \gamma_{h}^{1}} \int_{e}\left\{\nabla\left(\boldsymbol{U}_{I}-\boldsymbol{u}\right)\right\} \boldsymbol{n}_{e} \cdot\left[\boldsymbol{v}_{h}\right]\right| \leq C J_{0}\left(\boldsymbol{v}_{h}, \boldsymbol{v}_{h}\right)^{1 / 2} h^{k}|\boldsymbol{u}|_{k+1} .
$$

The first term vanishes on all segments of $\Gamma_{h}$ because $\left\{\nabla\left(\boldsymbol{U}-\boldsymbol{U}_{I}\right)\right\} \boldsymbol{n}_{e}$ belongs to $\mathbb{P}_{k-1}^{2}$ and $\boldsymbol{v}_{h}$ belongs to $\widetilde{\boldsymbol{X}_{h}}$. Thus it has the same structure as the term studied in Lemma 4.2

$$
\begin{aligned}
\left|\sum_{e \in \Gamma \cup \gamma_{h}^{1}} \int_{e}\left\{\nabla\left(\boldsymbol{U}-\boldsymbol{U}_{I}\right)\right\} \boldsymbol{n}_{e} \cdot\left[\boldsymbol{v}_{h}\right]\right| & \leq C J_{0}\left(\boldsymbol{v}_{h}, \boldsymbol{v}_{h}\right)^{1 / 2}\left\|\nabla\left(\boldsymbol{U}-\boldsymbol{U}_{I}\right)\right\|_{0, D^{12}} \\
& \leq C J_{0}\left(\boldsymbol{v}_{h}, \boldsymbol{v}_{h}\right)^{1 / 2} h^{k}\left(|\boldsymbol{u}|_{k+1}+\frac{1}{\mu}|p|_{k}\right) .
\end{aligned}
$$

The theorem is then obtained by combining these bounds with the triangle inequality and (5.5).

We now address the estimate for the velocity in the $L^{2}$ norm. The next theorem shows that the convergence is optimal in the mesh size in the symmetric case (Ca), but when $\epsilon^{*}=1$, we lose a power of $h$. The proof is written under the assumption that $\Omega$ is convex so that for any $\boldsymbol{g} \in L^{2}(\Omega)^{2}$, the solution $(\boldsymbol{\Phi}, \xi)$ of the dual problem

$$
\begin{aligned}
-\mu \Delta \boldsymbol{\Phi}+\nabla \xi & =\boldsymbol{g}, \quad \text { in } \Omega, \\
\nabla \cdot \boldsymbol{\Phi} & =0, \quad \text { in } \Omega, \\
\mathbf{\Phi} & =\mathbf{0}, \quad \text { on } \partial \Omega,
\end{aligned}
$$

belongs to $H^{2}(\Omega)^{2} \times H^{1}(\Omega)$ with continuous dependence on $\boldsymbol{g}$, .i.e,

$$
\|\boldsymbol{\Phi}\|_{2}+\frac{1}{\mu}\|\xi\|_{1} \leq \frac{C}{\mu}\|\boldsymbol{g}\|_{0} .
$$

The theorem handles both cases in order to show precisely where the nonsymmetric formulation loses the factor of $h$. However, the estimate (5.11) holds without the convexity assumption (see Lemma 6.2). 
Theorem 5.3. Assume that $\Omega$ is convex. Then, under the hypotheses of Theorem [5.1, there exists a constant $C$, independent of $h$ and $\mu$ such that

$$
\begin{gathered}
\|\boldsymbol{u}-\boldsymbol{U}\|_{0} \leq C h^{k+1}\left(|\boldsymbol{u}|_{k+1}+\frac{1}{\mu}|p|_{k}\right), \quad \epsilon^{*}=-1, \\
\|\boldsymbol{u}-\boldsymbol{U}\|_{0} \leq C h^{k}\left(|\boldsymbol{u}|_{k+1}+\frac{1}{\mu}|p|_{k}\right), \quad \epsilon^{*}=+1 .
\end{gathered}
$$

Proof. Consider the dual problem (5.6) -(5.8) with $\boldsymbol{g}=\boldsymbol{U}-\boldsymbol{u}$. Using Green's formula over each element of $\mathcal{E}_{h}$, we get

$$
\begin{aligned}
\|\boldsymbol{U}-\boldsymbol{u}\|_{0}^{2}= & \sum_{E} \int_{E}(\boldsymbol{U}-\boldsymbol{u}) \cdot(-\mu \Delta \boldsymbol{\Phi}+\nabla \xi) \\
= & \sum_{E} \int_{E} \mu \nabla(\boldsymbol{U}-\boldsymbol{u}): \nabla \boldsymbol{\Phi}-\sum_{E} \int_{\partial E}\left(\mu \nabla \boldsymbol{\Phi} \boldsymbol{n}_{E}\right) \cdot(\boldsymbol{U}-\boldsymbol{u}) \\
& -\sum_{E} \int_{E} \xi \nabla \cdot(\boldsymbol{U}-\boldsymbol{u})+\sum_{E} \int_{\partial E} \xi \boldsymbol{n}_{E} \cdot(\boldsymbol{U}-\boldsymbol{u}) .
\end{aligned}
$$

The regularity of $\boldsymbol{\Phi}$ and $\xi$ implies that $\left.[\mu \nabla \boldsymbol{\Phi}]\right|_{e} \boldsymbol{n}_{e}=\mathbf{0}$ and $\left.[\xi]\right|_{e}=0$ on each interior edge $e$. Thus, we have

$$
\|\boldsymbol{U}-\boldsymbol{u}\|_{0}^{2}=\sum_{E} \int_{E} \mu \nabla \boldsymbol{\Phi}: \nabla(\boldsymbol{U}-\boldsymbol{u})-\sum_{e \in \Gamma_{h} \cup \gamma_{h}^{1}} \int_{e}\{\mu \nabla \boldsymbol{\Phi}\} \boldsymbol{n}_{e} \cdot[\boldsymbol{U}-\boldsymbol{u}]+b(\boldsymbol{U}-\boldsymbol{u}, \xi) .
$$

By subtracting the orthogonality equations

$$
\begin{array}{ll}
\forall \boldsymbol{v}_{h} \in \boldsymbol{X}_{h}, & \mu\left(a\left(\boldsymbol{U}-\boldsymbol{u}, \boldsymbol{v}_{h}\right)+J_{0}\left(\boldsymbol{U}-\boldsymbol{u}, \boldsymbol{v}_{h}\right)\right)+b\left(\boldsymbol{v}_{h}, P-p\right)=0, \\
\forall q_{h} \in M_{h}, & b\left(\boldsymbol{U}-\boldsymbol{u}, q_{h}\right)=0,
\end{array}
$$

we obtain for any $\boldsymbol{v}_{h}$ in $\boldsymbol{X}_{h}$ and $q_{h}$ in $M_{h}$

$$
\begin{aligned}
\|\boldsymbol{U}-\boldsymbol{u}\|_{0}^{2}=\sum_{E} \int_{E} \mu \nabla\left(\boldsymbol{\Phi}-\boldsymbol{v}_{h}\right): \nabla(\boldsymbol{U}-\boldsymbol{u}) \\
\quad-\left(1+\epsilon^{*}\right) \sum_{e \in \Gamma_{h} \cup \gamma_{h}^{1}} \int_{e}\{\mu \nabla \boldsymbol{\Phi}\} \boldsymbol{n}_{e} \cdot[\boldsymbol{U}-\boldsymbol{u}] \\
+\epsilon^{*} \sum_{e \in \Gamma_{h} \cup \gamma_{h}^{1}} \int_{e}\left\{\mu \nabla\left(\boldsymbol{\Phi}-\boldsymbol{v}_{h}\right)\right\} \boldsymbol{n}_{e} \cdot[\boldsymbol{U}-\boldsymbol{u}] \\
\quad+\sum_{e \in \Gamma_{h} \cup \gamma_{h}^{1}} \int_{e}\{\mu \nabla(\boldsymbol{U}-\boldsymbol{u})\} \boldsymbol{n}_{e} \cdot\left[\boldsymbol{v}_{h}\right] \\
\quad-\mu J_{0}\left(\boldsymbol{U}-\boldsymbol{u}, \boldsymbol{v}_{h}\right)+b\left(\boldsymbol{U}-\boldsymbol{u}, \xi-q_{h}\right)-b\left(\boldsymbol{v}_{h}, P-p\right) \\
=A_{1}+A_{2}+\cdots+A_{7} .
\end{aligned}
$$

We choose $\boldsymbol{v}_{h}=\boldsymbol{P}_{h}(\boldsymbol{\Phi})$ and $q_{h}=r_{h}(\xi)$. First, observe that the properties of $\boldsymbol{\Phi}$ and 4.22) imply

$$
b\left(\boldsymbol{v}_{h}, P-p\right)=b\left(\boldsymbol{P}_{h}(\boldsymbol{\Phi})-\boldsymbol{\Phi}, P-p\right)+b(\boldsymbol{\Phi}, P-p)=b\left(\boldsymbol{P}_{h}(\boldsymbol{\Phi})-\boldsymbol{\Phi}, r_{h}(p)-p\right) .
$$


Moreover, comparing with (5.2), we obtain

$$
\begin{aligned}
& \left|b\left(\boldsymbol{P}_{h}(\boldsymbol{\Phi})-\boldsymbol{\Phi}, r_{h}(p)-p\right)\right| \\
& \leq \sqrt{2} \sum_{E \in \mathcal{E}_{h}}\left|\boldsymbol{P}_{h}(\boldsymbol{\Phi})-\boldsymbol{\Phi}\right|_{1, E}\left\|r_{h}(p)-p\right\|_{0, E} \\
& \quad+\hat{C}\left(\left\|r_{h}(p)-p\right\|_{0}^{2}+\sum_{E \in \mathcal{E}_{h}} h_{E}\left|r_{h}(p)-p\right|_{1, E}^{2}\right)^{1 / 2} J_{0}\left(\boldsymbol{P}_{h}(\boldsymbol{\Phi}), \boldsymbol{P}_{h}(\boldsymbol{\Phi})\right)^{1 / 2} \\
& \quad \leq \frac{C}{\mu} h^{k+1}|p|_{k}\|\boldsymbol{U}-\boldsymbol{u}\|_{0}
\end{aligned}
$$

owing to (4.23) with $s=2$ and (5.9). Similarly,

$$
\begin{aligned}
b\left(\boldsymbol{U}-\boldsymbol{u}, \xi-r_{h}(\xi)\right)= & -\sum_{E \in \mathcal{E}_{h}} \int_{E} \nabla \cdot\left(\boldsymbol{U}_{I}-\boldsymbol{u}\right)\left(\xi-r_{h}(\xi)\right) \\
& +\sum_{e \in \Gamma_{h}} \int_{e}\left\{\xi-r_{h}(\xi)\right\}[\boldsymbol{U}-\boldsymbol{u}] \cdot \boldsymbol{n}_{e} \\
& +\sum_{e \in \gamma_{h}^{1}} \int_{e}\left(\xi-r_{h}(\xi)\right)_{\gamma}[\boldsymbol{U}-\boldsymbol{u}] \cdot \boldsymbol{n}_{1},
\end{aligned}
$$

where $\boldsymbol{U}_{I}$ is an interpolant of $\boldsymbol{U}$ of degree $k$ in each element. Then comparing again the second term with (5.2) and (5.3), we obtain the same bound as above:

$$
\left|b\left(\boldsymbol{U}-\boldsymbol{u}, \xi-r_{h}(\xi)\right)\right| \leq C h^{k+1}\left(|\boldsymbol{u}|_{k+1}+\frac{1}{\mu}|p|_{k}\right)\|\boldsymbol{U}-\boldsymbol{u}\|_{0} .
$$

Next, the bound for $A_{1}$ is straightforward:

$$
A_{1} \leq C h^{k+1}\left(|\boldsymbol{u}|_{k+1}+\frac{1}{\mu}|p|_{k}\right)\|\boldsymbol{U}-\boldsymbol{u}\|_{0},
$$

$A_{3}$ is bounded as $T_{2}, A_{4}$ is bounded as $Q_{4}$ in Theorems 5.1 and [5.2, and considering that

$$
J_{0}\left(\boldsymbol{v}_{h}, \boldsymbol{v}_{h}\right)^{1 / 2}=J_{0}\left(\boldsymbol{P}_{h}(\boldsymbol{\Phi})-\boldsymbol{\Phi}, \boldsymbol{P}_{h}(\boldsymbol{\Phi})-\boldsymbol{\Phi}\right)^{1 / 2} \leq C h|\boldsymbol{\Phi}|_{2},
$$

it yields

$$
\left|A_{4}\right| \leq C h^{k+1}\left(|\boldsymbol{u}|_{k+1}+\frac{1}{\mu}|p|_{k}\right)\|\boldsymbol{U}-\boldsymbol{u}\|_{0}
$$

Similarly, by (5.12) and (5.1), we obtain

$$
J_{0}\left(\boldsymbol{U}-\boldsymbol{u}, \boldsymbol{v}_{h}\right) \leq J_{0}(\boldsymbol{U}-\boldsymbol{u}, \boldsymbol{U}-\boldsymbol{u})^{1 / 2} J_{0}\left(\boldsymbol{v}_{h}, \boldsymbol{v}_{h}\right)^{1 / 2} \leq C h^{k+1}\left(|\boldsymbol{u}|_{k+1}+\frac{1}{\mu}|p|_{k}\right) .
$$

It remains to derive a bound for $A_{2}$. This term vanishes as $\epsilon^{*}=-1$, and we can conclude. However, if $\epsilon^{*}=1$, this term is not zero, and this is where we shall lose a power of $h$. Arguing as for $Q_{5}$ in Theorem 5.2, we derive

$$
\left|A_{2}\right| \leq \mu C J_{0}(\boldsymbol{U}-\boldsymbol{u}, \boldsymbol{U}-\boldsymbol{u})^{1 / 2}\|\boldsymbol{\Phi}\|_{2},
$$


and with (5.9) this gives

$$
\left|A_{2}\right| \leq C J_{0}(\boldsymbol{U}-\boldsymbol{u}, \boldsymbol{U}-\boldsymbol{u})^{1 / 2}\|\boldsymbol{U}-\boldsymbol{u}\|_{0} \leq C h^{k}\left(|\boldsymbol{u}|_{k+1}+\frac{1}{\mu}|p|_{k}\right)\|\boldsymbol{U}-\boldsymbol{u}\|_{0} .
$$

This is the dominating term; all the other terms are of order $h^{k+1}$.

\section{NAVIER-STOKES PROBLEM}

We recall the Navier-Stokes system of equations (2.4), (2.2), 2.3):

$$
\begin{gathered}
-\mu \Delta \boldsymbol{u}+\boldsymbol{u} \cdot \nabla \boldsymbol{u}+\nabla p=\boldsymbol{f}, \quad \text { in } \Omega, \\
\nabla \cdot \boldsymbol{u}=0, \quad \text { in } \Omega, \\
\boldsymbol{u}=\mathbf{0}, \quad \text { on } \partial \Omega .
\end{gathered}
$$

We discretize the Stokes part of the equation with the left-hand side of (3.8), (3.9) and we address now the discretization of the nonlinear convection term $\boldsymbol{u} \cdot \nabla \boldsymbol{u}$. We shall use the following upwind discretization of $\boldsymbol{u} \cdot \nabla \boldsymbol{z}$ :

$$
\begin{aligned}
c(\boldsymbol{u} ; \boldsymbol{z}, \boldsymbol{\theta})=\sum_{E \in \mathcal{E}_{h}} & \left(\int_{E}(\boldsymbol{u} \cdot \nabla \boldsymbol{z}) \cdot \boldsymbol{\theta}+\int_{\partial E_{-}}\left|\{\boldsymbol{u}\} \cdot \boldsymbol{n}_{E}\right|\left(\boldsymbol{z}^{\mathrm{int}}-\boldsymbol{z}^{\mathrm{ext}}\right) \cdot \boldsymbol{\theta}^{\mathrm{int}}\right) \\
+ & \frac{1}{2} \sum_{E \in \mathcal{E}_{h}} \int_{E}(\nabla \cdot \boldsymbol{u}) \boldsymbol{z} \cdot \boldsymbol{\theta} \\
& -\frac{1}{2} \sum_{e \in \Gamma_{h} \cup \gamma_{h}^{1}} \int_{e}[\boldsymbol{u}] \cdot \boldsymbol{n}_{e}\{\boldsymbol{z} \cdot \boldsymbol{\theta}\}, \quad \forall \boldsymbol{u}, \boldsymbol{z}, \boldsymbol{\theta} \in \boldsymbol{X},
\end{aligned}
$$

where

$$
\partial E_{-}=\left\{\boldsymbol{x} \in \partial E:\{\boldsymbol{u}\} \cdot \boldsymbol{n}_{E}<0\right\}
$$

and the superscript int (resp. ext) refers to the trace of the function on a side of $E$ coming from the interior of $E$ (resp. coming from the exterior of $E$ on that side). When the side of $E$ belongs to $\partial \Omega$, then we take the exterior trace to be zero. The first three terms in the definition of $c$ were introduced in [17. for solving transport problems; the last term is chosen so that $c$ satisfies (6.6), which ensures its positivity. It is easy to see that, when $\boldsymbol{u}, \boldsymbol{z}, \boldsymbol{\theta} \in H_{0}^{1}(\Omega)^{2}, c$ reduces to

$$
c(\boldsymbol{u} ; \boldsymbol{z}, \boldsymbol{\theta})=\int_{\Omega}(\boldsymbol{u} \cdot \nabla \boldsymbol{z}) \cdot \boldsymbol{\theta}+\frac{1}{2} \int_{\Omega}(\nabla \cdot \boldsymbol{u}) \boldsymbol{z} \cdot \boldsymbol{\theta} .
$$

Then we discretize (2.4), (2.2), (2.3) by: find $(\boldsymbol{U}, P) \in \boldsymbol{X}_{h} \times M_{h}$ such that

$$
\begin{gathered}
\forall \boldsymbol{v}_{h} \in \boldsymbol{X}_{h}, \quad \mu\left(a\left(\boldsymbol{U}, \boldsymbol{v}_{h}\right)+J_{0}\left(\boldsymbol{U}, \boldsymbol{v}_{h}\right)\right)+c\left(\boldsymbol{U} ; \boldsymbol{U}, \boldsymbol{v}_{h}\right)+b\left(\boldsymbol{v}_{h}, P\right)=\int_{\Omega} \boldsymbol{f} \cdot \boldsymbol{v}_{h}, \\
\forall q_{h} \in M_{h}, \quad b\left(\boldsymbol{U}, q_{h}\right)=0 .
\end{gathered}
$$

In view of (6.2), the argument of Lemma 3.6 shows that every solution of the Navier-Stokes problem is also a solution of (6.3), (6.4) and conversely. The first lemma shows that $c$ has a nice "integration by parts" property. 
Lemma 6.1. The trilinear form $c$ defined by (6.1) satisfies the following "integration by parts" for all $\boldsymbol{u}, \boldsymbol{z}, \boldsymbol{\theta}$ in $\boldsymbol{X}$ :

$$
\begin{aligned}
c(\boldsymbol{u} ; \boldsymbol{z}, \boldsymbol{\theta})= & -\sum_{E \in \mathcal{E}_{h}}\left(\int_{E}(\boldsymbol{u} \cdot \nabla \boldsymbol{\theta}) \cdot \boldsymbol{z}+\frac{1}{2} \int_{E}(\nabla \cdot \boldsymbol{u}) \boldsymbol{z} \cdot \boldsymbol{\theta}\right) \\
& +\frac{1}{2} \sum_{e \in \Gamma_{h} \cup \gamma_{h}^{1}} \int_{e}[\boldsymbol{u}] \cdot \boldsymbol{n}_{e}\{\boldsymbol{z} \cdot \boldsymbol{\theta}\} \\
& -\sum_{E \in \mathcal{E}_{h}} \int_{\partial E_{-}}\left|\{\boldsymbol{u}\} \cdot \boldsymbol{n}_{E}\right| \boldsymbol{z}^{\mathrm{ext}} \cdot\left(\boldsymbol{\theta}^{\mathrm{int}}-\boldsymbol{\theta}^{\mathrm{ext}}\right)+\int_{\Gamma_{+}}|\boldsymbol{u} \cdot \boldsymbol{n}| \boldsymbol{z} \cdot \boldsymbol{\theta},
\end{aligned}
$$

where $\Gamma_{+}$is the subset of $\partial \Omega$ where $\boldsymbol{u} \cdot \boldsymbol{n}>0$. In particular, if $\boldsymbol{z}=\boldsymbol{\theta}$, we obtain

$$
c(\boldsymbol{u} ; \boldsymbol{z}, \boldsymbol{z})=\frac{1}{2} \sum_{E \in \mathcal{E}_{h}} \int_{\partial E_{-}}\left|\{\boldsymbol{u}\} \cdot \boldsymbol{n}_{E}\right|\left\|\boldsymbol{z}^{\mathrm{ext}}-\boldsymbol{z}^{\mathrm{int}}\right\|^{2}+\frac{1}{2} \int_{\Gamma_{+}}|\boldsymbol{u} \cdot \boldsymbol{n}|\|\boldsymbol{z}\|^{2} \geq 0 .
$$

Proof. Noting that $\nabla \cdot\left(\boldsymbol{u} z_{i}\right)=(\nabla \cdot \boldsymbol{u}) z_{i}+\boldsymbol{u} \cdot \nabla z_{i}$, we can write

$$
\begin{aligned}
\int_{E}(\boldsymbol{u} \cdot \nabla \boldsymbol{z}) \cdot \boldsymbol{\theta} & =\int_{E} \nabla \cdot(\boldsymbol{z} \otimes \boldsymbol{u}) \cdot \boldsymbol{\theta}-\int_{E}(\nabla \cdot \boldsymbol{u}) \boldsymbol{z} \cdot \boldsymbol{\theta} \\
& =-\int_{E}(\boldsymbol{u} \cdot \nabla \boldsymbol{\theta}) \cdot \boldsymbol{z}+\int_{\partial E}\left(\boldsymbol{u}^{\mathrm{int}} \cdot \boldsymbol{n}_{E}\right) \boldsymbol{z}^{\mathrm{int}} \cdot \boldsymbol{\theta}^{\mathrm{int}}-\int_{E}(\nabla \cdot \boldsymbol{u}) \boldsymbol{z} \cdot \boldsymbol{\theta} .
\end{aligned}
$$

Therefore

$$
\begin{aligned}
c(\boldsymbol{u} ; \boldsymbol{z}, \boldsymbol{\theta})= & -\sum_{E \in \mathcal{E}_{h}}\left(\int_{E}(\boldsymbol{u} \cdot \nabla \boldsymbol{\theta}) \cdot \boldsymbol{z}+\frac{1}{2} \int_{E}(\nabla \cdot \boldsymbol{u}) \boldsymbol{z} \cdot \boldsymbol{\theta}\right) \\
& +\sum_{E \in \mathcal{E}_{h}} \int_{\partial E_{-}}\left|\{\boldsymbol{u}\} \cdot \boldsymbol{n}_{E}\right|\left(\boldsymbol{z}^{\mathrm{int}}-\boldsymbol{z}^{\mathrm{ext}}\right) \cdot \boldsymbol{\theta}^{\mathrm{int}} \\
& -\frac{1}{2} \sum_{e \in \Gamma_{h} \cup \gamma_{h}^{1}} \int_{e}[\boldsymbol{u}] \cdot \boldsymbol{n}_{e}\{\boldsymbol{z} \cdot \boldsymbol{\theta}\} \\
& +\sum_{E \in \mathcal{E}_{h}} \int_{\partial E}\left(\boldsymbol{u}^{\mathrm{int}} \cdot \boldsymbol{n}_{E}\right) \boldsymbol{z}^{\mathrm{int}} \cdot \boldsymbol{\theta}^{\mathrm{int}} .
\end{aligned}
$$

In the last integral, let us split $\left.\boldsymbol{u}^{\text {int }}\right|_{\partial E}$ into

$$
\boldsymbol{u}^{\text {int }}=\{\boldsymbol{u}\}+\frac{1}{2}\left(\boldsymbol{u}^{\mathrm{int}}-\boldsymbol{u}^{\mathrm{ext}}\right) .
$$

Therefore

$\int_{\partial E}\left(\boldsymbol{u}^{\mathrm{int}} \cdot \boldsymbol{n}_{E}\right) \boldsymbol{z}^{\mathrm{int}} \cdot \boldsymbol{\theta}^{\mathrm{int}}=\int_{\partial E}\{\boldsymbol{u}\} \cdot \boldsymbol{n}_{E} \boldsymbol{z}^{\mathrm{int}} \cdot \boldsymbol{\theta}^{\mathrm{int}}+\frac{1}{2} \int_{\partial E}\left(\boldsymbol{u}^{\mathrm{int}}-\boldsymbol{u}^{\mathrm{ext}}\right) \cdot \boldsymbol{n}_{E} \boldsymbol{z}^{\mathrm{int}} \cdot \boldsymbol{\theta}^{\mathrm{int}}$.

We now sum the last integral over all $E$ and consider the contribution of this sum to one interior edge $e$. Assume that $e$ is shared by two triangles $E^{1}$ and $E^{2}$, with exterior normal $\boldsymbol{n}_{1}$ and $\boldsymbol{n}_{2}$; we obtain

$$
\begin{gathered}
\left.\left.\left.\frac{1}{2} \int_{e}\left(\left.\boldsymbol{u}\right|_{E^{1}}-\left.\boldsymbol{u}\right|_{E^{2}}\right) \cdot \boldsymbol{n}_{1} \boldsymbol{z}\right|_{E^{1}} \cdot \boldsymbol{\theta}\right|_{E^{1}}+\left.\left.\frac{1}{2} \int_{e}\left(\left.\boldsymbol{u}\right|_{E^{2}}-\left.\boldsymbol{u}\right|_{E^{1}}\right) \cdot \boldsymbol{n}_{2} \boldsymbol{z}\right|_{E^{2}} \cdot \boldsymbol{\theta}\right|_{E^{2}}\right) \\
=\int_{e}[\boldsymbol{u}] \cdot \boldsymbol{n}_{e}\{\boldsymbol{z} \cdot \boldsymbol{\theta}\} .
\end{gathered}
$$


The same formula is valid when $e$ is shared on one side by more than one triangle. It is also valid when $e \subset \partial \Omega$ because the jump and average coincide with the trace. Hence

$$
\begin{aligned}
c(\boldsymbol{u} ; \boldsymbol{z}, \boldsymbol{\theta})= & -\sum_{E \in \mathcal{E}_{h}}\left(\int_{E}(\boldsymbol{u} \cdot \nabla \boldsymbol{\theta}) \cdot \boldsymbol{z}+\frac{1}{2} \int_{E}(\nabla \cdot \boldsymbol{u}) \boldsymbol{z} \cdot \boldsymbol{\theta}\right) \\
& +\sum_{E \in \mathcal{E}_{h}} \int_{\partial E_{-}}\left|\{\boldsymbol{u}\} \cdot \boldsymbol{n}_{E}\right|\left(\boldsymbol{z}^{\mathrm{int}}-\boldsymbol{z}^{\mathrm{ext}}\right) \cdot \boldsymbol{\theta}^{\mathrm{int}} \\
& +\frac{1}{2} \sum_{e \in \Gamma_{h} \cup \gamma_{h}^{1}} \int_{e}[\boldsymbol{u}] \cdot \boldsymbol{n}_{e}\{\boldsymbol{z} \cdot \boldsymbol{\theta}\} \\
& +\sum_{E \in \mathcal{E}_{h}} \int_{\partial E}\left(\{\boldsymbol{u}\} \cdot \boldsymbol{n}_{E}\right) \boldsymbol{z}^{\mathrm{int}} \cdot \boldsymbol{\theta}^{\mathrm{int}} .
\end{aligned}
$$

But since $\{\boldsymbol{u}\}$ is now continuous across the edges, the argument used in [17] gives

$$
\begin{gathered}
\sum_{E \in \mathcal{E}_{h}} \int_{\partial E_{-}}\left|\{\boldsymbol{u}\} \cdot \boldsymbol{n}_{E}\right|\left(\boldsymbol{z}^{\mathrm{int}}-\boldsymbol{z}^{\mathrm{ext}}\right) \cdot \boldsymbol{\theta}^{\mathrm{int}}+\sum_{E \in \mathcal{E}_{h}} \int_{\partial E}\left(\{\boldsymbol{u}\} \cdot \boldsymbol{n}_{E}\right) \boldsymbol{z}^{\mathrm{int}} \cdot \boldsymbol{\theta}^{\mathrm{int}} \\
=\sum_{E \in \mathcal{E}_{h}} \int_{\partial E_{-}}\left|\{\boldsymbol{u}\} \cdot \boldsymbol{n}_{E}\right| \boldsymbol{z}^{\mathrm{ext}} \cdot\left(\boldsymbol{\theta}^{\mathrm{ext}}-\boldsymbol{\theta}^{\mathrm{int}}\right)+\int_{\Gamma_{+}} \boldsymbol{u} \cdot \boldsymbol{n} \boldsymbol{z} \cdot \boldsymbol{\theta} .
\end{gathered}
$$

This gives (6.5). Then if we take $\boldsymbol{\theta}=\boldsymbol{z}$ and add (6.1) to (6.5), we recover (6.6).

The next lemma gives an $L^{p}$ estimate for functions in $X_{h}$, in terms of the norm [\|. ]. It is stated for any function in the space

$$
H^{1}\left(\mathcal{E}_{h}\right)=\left\{v \in L^{2}(\Omega): \forall E \in \mathcal{E}_{h},\left.\quad v\right|_{E} \in H^{1}(E)\right\}
$$

Lemma 6.2. Assume that the triangulation satisfies (3.1) and (4.2). Then for each real number $p \in[2, \infty)$ there exists a constant $C(p)$, independent of $h$, such that

$$
\forall v \in H^{1}\left(\mathcal{E}_{h}\right), \quad\|v\|_{L^{p}(\Omega)} \leq C(p) \llbracket v \| .
$$

Proof. If $v$ were in $H_{0}^{1}(\Omega)$, then (6.8) would follow from Sobolev's imbedding (2.6). Since $v$ does not belong to $H_{0}^{1}(\Omega)$, the idea of the proof is to associate with $v$ a suitable function $v(h)$ whose gradient is closely related to $\nabla v$ in each $E$ and to study the difference $v-v(h)$. Thus, we define $v(h)$ in $H_{0}^{1}(\Omega)$ by

$$
\forall w \in H_{0}^{1}(\Omega), \quad \int_{\Omega} \nabla v(h) \cdot \nabla w=\sum_{E \in \mathcal{E}_{h}} \int_{E} \nabla v \cdot \nabla w .
$$

Then

$$
\|\nabla v(h)\|_{0} \leq\|\nabla v\|_{0}
$$

Now the proof proceeds by duality: we write

$$
\|v(h)-v\|_{L^{p}(\Omega)}=\sup _{g \in L^{p^{\prime}}(\Omega)} \frac{\int_{\Omega}(v(h)-v) g}{\|g\|_{L^{p^{\prime}}(\Omega)}},
$$

where $p^{\prime}$ is the dual exponent of $p: \frac{1}{p}+\frac{1}{p^{\prime}}=1$. For a fixed $g$ in $L^{p^{\prime}}(\Omega)$, let $\varphi \in H_{0}^{1}(\Omega)$ be the solution of

$$
-\Delta \varphi=g, \quad \text { in } \Omega,\left.\quad \varphi\right|_{\partial \Omega}=0
$$


When $p>4$, then $p^{\prime}<\frac{4}{3}$ and it follows from [14] that $\varphi \in W^{2, p^{\prime}}(\Omega)$ with

$$
\|\varphi\|_{W^{2, p^{\prime}}(\Omega)} \leq C_{1}(p)\|g\|_{L^{p^{\prime}}(\Omega)} .
$$

When $p \leq 4$, then $p^{\prime} \geq \frac{4}{3}$ and $g$ belongs always to $L^{4 / 3}(\Omega)$. Therefore, we also have $\varphi \in W^{2,4 / 3}(\Omega)$ with

$$
\|\varphi\|_{W^{2,4 / 3}(\Omega)} \leq C_{1}(4)\|g\|_{L^{4 / 3}(\Omega)} \leq|\Omega|^{\frac{1}{p}-\frac{1}{4}} C_{1}(4)\|g\|_{L^{p^{\prime}}(\Omega)}=C_{2}(p)\|g\|_{0, L^{p^{\prime}}(\Omega)} .
$$

Now (6.11) implies that

$$
\begin{aligned}
\int_{\Omega}(v(h)-v) g & =-\int_{\Omega} \Delta \varphi(v(h)-v)=-\sum_{E \in \mathcal{E}_{h}} \int_{E} \Delta \varphi(v(h)-v) \\
& =\sum_{E \in \mathcal{E}_{h}} \int_{E} \nabla \varphi \cdot \nabla(v(h)-v)-\sum_{E \in \mathcal{E}_{h}} \int_{\partial E} \nabla \varphi \cdot \boldsymbol{n}_{E}(v(h)-v) \\
& =-\sum_{e \in \Gamma_{h} \cup \gamma_{h}^{1}} \int_{e}\{\nabla \varphi\} \cdot \boldsymbol{n}_{e}[v(h)-v],
\end{aligned}
$$

owing to (6.9) and the regularity of $\varphi$. Let $p>4$; by the trace theorem and the regularity properties (3.1) and (4.2) of $\mathcal{E}_{h}$, we have

$$
\left|\int_{\Omega}(v(h)-v) g\right| \leq C_{3}(p)\left(|\varphi|_{1, \Omega}+h^{2 / p}|\varphi|_{W^{2, p^{\prime}}(\Omega)}\right) J_{0}(v, v)^{1 / 2} .
$$

If $p \leq 4$, we use the fact that $\varphi \in W^{2,4 / 3}(\Omega)$ and (6.14) is replaced by

$$
\left|\int_{\Omega}(v(h)-v) g\right| \leq C_{3}(4)\left(|\varphi|_{1, \Omega}+h^{1 / 2}|\varphi|_{W^{2,4 / 3}(\Omega)}\right) J_{0}(v, v)^{1 / 2} .
$$

With (6.12) and (6.13), this becomes

$$
\left|\int_{\Omega}(v(h)-v) g\right| \leq C_{4}(p)\|g\|_{L^{p^{\prime}}(\Omega)} J_{0}(v, v)^{1 / 2} .
$$

Therefore

$$
\|v(h)-v\|_{L^{p}(\Omega)} \leq C_{4}(p) J_{0}(v, v)^{1 / 2},
$$

and hence by virtue of (2.6) and (6.10), we have (6.8) with

$$
C(p)=\left(C_{4}(p)^{2}+S_{p}^{2}\right)^{1 / 2} .
$$

Lemmas 6.1, 6.2 and Theorem 4.5 enable us to prove the existence of discrete solutions and a priori estimates.

Proposition 6.3. Assume that the triangulation satisfies (3.1) and Hypotheses H1 and $\mathrm{H} 2$. Then, if $\mathrm{H} 3$ holds, for any $\boldsymbol{f}$ in $L^{4 / 3}(\Omega)^{2}$ and $\mu>0$, the discrete NavierStokes problem (6.3), (6.4) has at least one solution $(\boldsymbol{U}, P)$ in $\boldsymbol{X}_{h} \times M_{h}$, and each solution satisfies the a priori estimates

$$
\begin{gathered}
\sum_{E \in \mathcal{E}_{h}} \int_{\partial E_{-}}\left|\{\boldsymbol{U}\} \cdot \boldsymbol{n}_{E}\right|\left\|\boldsymbol{U}^{\mathrm{int}}-\boldsymbol{U}^{\mathrm{ext}}\right\|^{2}+\int_{\Gamma_{+}} \mid \boldsymbol{U} \cdot \boldsymbol{n}\|\boldsymbol{U}\|^{2} \leq \frac{2}{\mu K} C(4)^{2}\|\boldsymbol{f}\|_{L^{4 / 3}(\Omega)}^{2}, \\
\|P\|_{0} \leq C\left(\|\boldsymbol{f}\|_{L^{4 / 3}(\Omega)}+\llbracket \boldsymbol{U} \rrbracket^{2}\right),
\end{gathered}
$$


where $C(4)$ is the constant of Lemma 6.2 with exponent 4 and $C$ is another constant that depends on $\beta^{*}$ (see (4.8)) but is independent of $h$ and $\mu$.

We skip the proof because the existence of $\boldsymbol{U}$ is a straightforward application of Brouwer's Fixed Point Theorem in the space $\boldsymbol{V}_{h}$. It is made possible by (6.6) and (6.8) with exponent 4. Then the existence of $P$ follows from Theorem 4.5.

Now, we turn to error estimates. Let $(\boldsymbol{U}, P)$ be a solution of (6.3), 6.4), let $(\boldsymbol{u}, p)$ be a solution of (2.4), (2.2), (2.3), let $\boldsymbol{U}_{I}=\boldsymbol{P}_{h}(\boldsymbol{u})$ be the operator defined in Corollary 4.8 and let $P_{I}=r_{h}(p)$ be the operator defined in (3.10). Then taking the difference between (6.3) and the same equation satisfied by the exact solution, using (4.22) and setting $\boldsymbol{\chi}=\boldsymbol{U}-\boldsymbol{U}_{I}, \xi=P-P_{I}$, we obtain the following equation:

$$
\begin{array}{r}
\mu\left(a(\boldsymbol{\chi}, \boldsymbol{\chi})+J_{0}(\boldsymbol{\chi}, \boldsymbol{\chi})\right)+c\left(\boldsymbol{U}_{I} ; \boldsymbol{\chi}, \boldsymbol{\chi}\right)+c(\boldsymbol{\chi} ; \boldsymbol{U}, \boldsymbol{\chi})=\mu\left(a\left(\boldsymbol{u}-\boldsymbol{U}_{I}, \boldsymbol{\chi}\right)+J_{0}\left(\boldsymbol{u}-\boldsymbol{U}_{I}, \boldsymbol{\chi}\right)\right) \\
+b\left(\boldsymbol{\chi}, p-P_{I}\right)+c\left(\boldsymbol{u}-\boldsymbol{U}_{I} ; \boldsymbol{U}_{I}, \boldsymbol{\chi}\right)+c\left(\boldsymbol{u} ; \boldsymbol{u}-\boldsymbol{U}_{I}, \boldsymbol{\chi}\right) .
\end{array}
$$

Owing to (6.6), the left-hand side is bounded below by

$K \mu \llbracket \chi \chi \rrbracket^{2}+\frac{1}{2} \sum_{E \in \mathcal{E}_{h}} \int_{\partial E_{-}}\left|\left\{\boldsymbol{U}_{I}\right\} \cdot \boldsymbol{n}_{e}\right|\left\|\boldsymbol{\chi}^{\mathrm{ext}}-\boldsymbol{\chi}^{\mathrm{int}}\right\|^{2}+\frac{1}{2} \int_{\Gamma_{+}}|\boldsymbol{U} \cdot \boldsymbol{n}|\|\boldsymbol{\chi}\|^{2}+c(\boldsymbol{\chi} ; \boldsymbol{U}, \boldsymbol{\chi})$.

Therefore, we must find an upper bound for $c(\boldsymbol{\chi} ; \boldsymbol{U}, \boldsymbol{\chi})$.

Lemma 6.4. Assume that the mesh satisfies (3.1) and (4.2). There exists a constant $C$ and for each $r>2$, there exists a constant $C_{r}$, both independent of $h$, such that

$$
\begin{aligned}
\forall \boldsymbol{u}_{h} \in \boldsymbol{V}_{h}, \forall \boldsymbol{v}_{h}, \boldsymbol{w}_{h} \in \boldsymbol{X}_{h}, \quad\left|c\left(\boldsymbol{u}_{h} ; \boldsymbol{v}_{h}, \boldsymbol{w}_{h}\right)\right| \leq C_{r} h^{2 / r} \llbracket \llbracket \boldsymbol{u}_{h} \rrbracket \llbracket \llbracket \boldsymbol{v}_{h} \rrbracket \llbracket \mid \boldsymbol{w}_{h} \rrbracket \\
+C\left\|\boldsymbol{w}_{h}\right\|_{L^{4}(\Omega)}\left(\left\|\boldsymbol{u}_{h}\right\|_{L^{4}(\Omega)} \llbracket \mid \boldsymbol{v}_{h} \rrbracket+\left\|\boldsymbol{v}_{h}\right\|_{L^{4}(\Omega)} J_{0}\left(\boldsymbol{u}_{h}, \boldsymbol{u}_{h}\right)^{1 / 2}\right) .
\end{aligned}
$$

Proof. Observe that

$$
\begin{aligned}
c\left(\boldsymbol{u}_{h} ; \boldsymbol{v}_{h}, \boldsymbol{w}_{h}\right)= & \sum_{E \in \mathcal{E}_{h}}\left(\int_{E}\left(\boldsymbol{u}_{h} \cdot \nabla \boldsymbol{v}_{h}\right) \cdot \boldsymbol{w}_{h}+\int_{\partial E_{-}}\left|\left\{\boldsymbol{u}_{h}\right\} \cdot \boldsymbol{n}_{e}\right|\left(\boldsymbol{v}_{h}^{\mathrm{int}}-\boldsymbol{v}_{h}^{\mathrm{ext}}\right) \cdot \boldsymbol{w}_{h}^{\mathrm{int}}\right) \\
& -\frac{1}{2} b\left(\boldsymbol{u}_{h}, \boldsymbol{v}_{h} \cdot \boldsymbol{w}_{h}\right)+\frac{\epsilon}{4} \sum_{e \in \gamma_{h}^{1}} \int_{e}\left[\boldsymbol{u}_{h}\right] \cdot \boldsymbol{n}_{1}\left[\boldsymbol{v}_{h} \cdot \boldsymbol{w}_{h}\right] .
\end{aligned}
$$

To bound the third term, we use an argument of Girault and Lions [10. By the definition of $\boldsymbol{V}_{h}$, we can write in particular

$$
b\left(\boldsymbol{u}_{h}, \boldsymbol{v}_{h} \cdot \boldsymbol{w}_{h}\right)=b\left(\boldsymbol{u}_{h}, \boldsymbol{v}_{h} \cdot \boldsymbol{w}_{h}-q_{h}\right),
$$

where in each $E, q_{h}$ is the scalar product of two constant vectors $\boldsymbol{c}_{1} \cdot \boldsymbol{c}_{2}$ :

$$
\left.\left(\boldsymbol{v}_{h} \cdot \boldsymbol{w}_{h}-q_{h}\right)\right|_{E}=\left.\left(\boldsymbol{v}_{h} \cdot \boldsymbol{w}_{h}-\boldsymbol{c}_{1} \cdot \boldsymbol{c}_{2}\right)\right|_{E}=\left(\boldsymbol{v}_{h}-\boldsymbol{c}_{1}\right) \cdot \boldsymbol{w}_{h}+\boldsymbol{c}_{1} \cdot\left(\boldsymbol{w}_{h}-\boldsymbol{c}_{2}\right) .
$$

Then arguing as in Corollary 4.8, we derive

$$
\left|b\left(\boldsymbol{u}_{h}, \boldsymbol{v}_{h} \cdot \boldsymbol{w}_{h}\right)\right| \leq C \llbracket \boldsymbol{u}_{h} \rrbracket\left(\sum_{E \in \mathcal{E}_{h}}\left\|\left(\boldsymbol{v}_{h}-\boldsymbol{c}_{1}\right) \cdot \boldsymbol{w}_{h}\right\|_{0, E}^{2}+\left\|\boldsymbol{c}_{1} \cdot\left(\boldsymbol{w}_{h}-\boldsymbol{c}_{2}\right)\right\|_{0, E}^{2}\right)^{1 / 2} .
$$

Let us choose

$$
\boldsymbol{c}_{1}=\frac{1}{|E|} \int_{E} \boldsymbol{v}_{h}, \quad \boldsymbol{c}_{2}=\frac{1}{|E|} \int_{E} \boldsymbol{w}_{h},
$$


and for some number $r>2$, let us write

$\left\|\left(\boldsymbol{v}_{h}-\boldsymbol{c}_{1}\right) \cdot \boldsymbol{w}_{h}\right\|_{0, E} \leq\left\|\boldsymbol{v}_{h}-\boldsymbol{c}_{1}\right\|_{L^{r}(E)}\left\|\boldsymbol{w}_{h}\right\|_{L^{\frac{2 r}{r-2}(E)}} \leq C|E|^{1 / r}\left\|\nabla \boldsymbol{v}_{h}\right\|_{0, E}\left\|\boldsymbol{w}_{h}\right\|_{L^{\frac{2 r}{r-2}(E)}}$.

Then summing over all $E$ and applying (6.8) to each component of $\boldsymbol{w}_{h}$ with exponent $(2 r) /(r-2)$, we obtain for any fixed $r>2$ :

$$
\left(\sum_{E \in \mathcal{E}_{h}}\left\|\left(\boldsymbol{v}_{h}-\boldsymbol{c}_{1}\right) \cdot \boldsymbol{w}_{h}\right\|_{0, E}^{2}\right)^{1 / 2} \leq C h^{2 / r}\left\|\nabla \boldsymbol{v}_{h}\right\|_{0} \llbracket \| \boldsymbol{w}_{h} \rrbracket
$$

Similarly,

$$
\left(\sum_{E \in \mathcal{E}_{h}}\left\|\boldsymbol{c}_{1} \cdot\left(\boldsymbol{w}_{h}-\boldsymbol{c}_{2}\right)\right\|_{0, E}^{2}\right)^{1 / 2} \leq C h^{2 / r}\left\|\nabla \boldsymbol{w}_{h}\right\|_{0} \llbracket \boldsymbol{v}_{h} \rrbracket .
$$

Therefore, for each $r>2$, there exists a constant $C_{r}$, independent of $h$, such that

$$
\left|b\left(\boldsymbol{u}_{h}, \boldsymbol{v}_{h} \cdot \boldsymbol{w}_{h}\right)\right| \leq C_{r} h^{2 / r} \llbracket \mid \boldsymbol{u}_{h} \|\left[\| \boldsymbol { v } _ { h } \| \left[\left\|\boldsymbol{w}_{h}\right\| .\right.\right.
$$

For the second term, we have

$$
\left|\sum_{E \in \mathcal{E}_{h}} \int_{\partial E_{-}}\right|\left\{\boldsymbol{u}_{h}\right\} \cdot \boldsymbol{n}_{e}\left|\left(\boldsymbol{v}_{h}^{\text {int }}-\boldsymbol{v}_{h}^{\text {ext }}\right) \cdot \boldsymbol{w}_{h}^{\text {int }}\right| \leq C J_{0}\left(\boldsymbol{v}_{h}, \boldsymbol{v}_{h}\right)^{1 / 2}\left\|\boldsymbol{u}_{h}\right\|_{L^{4}(\Omega)}\left\|\boldsymbol{w}_{h}\right\|_{L^{4}(\Omega)} \text {. }
$$

The last term is bounded as follows:

$$
\left|\sum_{e \in \gamma_{h}^{1}} \int_{e}\left[\boldsymbol{u}_{h}\right] \cdot \boldsymbol{n}_{1}\left[\boldsymbol{v}_{h} \cdot \boldsymbol{w}_{h}\right]\right| \leq C J_{0}\left(\boldsymbol{u}_{h}, \boldsymbol{u}_{h}\right)^{1 / 2}\left\|\boldsymbol{v}_{h}\right\|_{L^{4}(\Omega)}\left\|\boldsymbol{w}_{h}\right\|_{L^{4}(\Omega)},
$$

and the first term has the straightforward bound

$$
\left|\sum_{E \in \mathcal{E}_{h}} \int_{E}\left(\boldsymbol{u}_{h} \cdot \nabla \boldsymbol{v}_{h}\right) \cdot \boldsymbol{w}_{h}\right| \leq\left\|\nabla \boldsymbol{v}_{h}\right\|_{0}\left\|\boldsymbol{u}_{h}\right\|_{L^{4}(\Omega)}\left\|\boldsymbol{w}_{h}\right\|_{L^{4}(\Omega)} .
$$

Then (6.18) follows by collecting all these bounds.

Remark 6.5. When the first argument of $c$ belongs to $\boldsymbol{V}$ (instead of $\boldsymbol{V}_{h}$ ), then only the first two terms in the expansion of $c$ remain and

$\left|c\left(\boldsymbol{u} ; \boldsymbol{v}_{h}, \boldsymbol{w}_{h}\right)\right| \leq\left\|\boldsymbol{w}_{h}\right\|_{L^{4}(\Omega)}\left(\|\boldsymbol{u}\|_{L^{4}(\Omega)}\left\|\nabla \boldsymbol{v}_{h}\right\|_{0}+C J_{0}\left(\boldsymbol{v}_{h}, \boldsymbol{v}_{h}\right)^{1 / 2}\left(\|\boldsymbol{u}\|_{L^{4}(\Omega)}+h^{1 / 2}|\boldsymbol{u}|_{1}\right)\right)$, where the constant $C$ is independent of $h$. When the first argument of $c$ belongs to $\boldsymbol{V}+\boldsymbol{V}_{h}$, then (6.18) is replaced by

$$
\begin{array}{r}
\left|c\left(\boldsymbol{u}-\boldsymbol{u}_{h} ; \boldsymbol{v}_{h}, \boldsymbol{w}_{h}\right)\right| \leq\left\|\boldsymbol{w}_{h}\right\|_{L^{4}(\Omega)}\left(C \left[\left\|\boldsymbol{u}-\boldsymbol{u}_{h}\right\|\left[\left\|\boldsymbol{v}_{h}\right\|+\left\|\boldsymbol{u}-\boldsymbol{u}_{h}\right\|_{L^{4}(\Omega)}\left\|\nabla \boldsymbol{v}_{h}\right\|_{0}\right)\right.\right. \\
+C_{r} h^{2 / r}\left[\| \boldsymbol { u } - \boldsymbol { u } _ { h } \| \left[\| \boldsymbol { v } _ { h } \| \left[\left\|\boldsymbol{w}_{h}\right\| .\right.\right.\right.
\end{array}
$$

As is usual for the Navier-Stokes equations, we introduce the quantity

$$
N(h)=\sup _{\boldsymbol{v}_{h}, \boldsymbol{w}_{h} \in \boldsymbol{V}_{h}} \frac{c\left(\boldsymbol{w}_{h} ; \boldsymbol{v}_{h}, \boldsymbol{w}_{h}\right)}{\left\|\boldsymbol{v}_{h}\right\|\left[\boldsymbol{w}_{h} \|^{2}\right.} .
$$

According to Lemmas 6.1 and 6.2, $N(h)$ is bounded by a constant $N$ independent of $h$. It is easy to check that the discrete Navier-Stokes problem (6.3), (6.4) has a unique solution if the data satisfy

$$
\frac{N}{K^{2} \mu^{2}} C(4)\|\boldsymbol{f}\|_{L^{4 / 3}(\Omega)}<1,
$$

where $C(4)$ is the constant of (6.8) with exponent 4 . We have the following error estimates. 
Theorem 6.6. Under the assumptions of Theorem 5.1 and if the data $\boldsymbol{f}$ and $\mu$ satisfy:

$$
\frac{N}{K^{2} \mu^{2}} C(4)\|\boldsymbol{f}\|_{L^{4 / 3}(\Omega)} \leq \frac{1}{2},
$$

then, the solution $(\boldsymbol{U}, P)$ of (6.3), (6.4) satisfies the following a priori error estimates:

$$
\begin{gathered}
\| \boldsymbol{u}-\boldsymbol{U} \rrbracket \leq C h^{k}\left(\left(1+\frac{1}{\mu^{2}}\right)|\boldsymbol{u}|_{k+1}+\frac{1}{\mu}|p|_{k}\right), \\
\|p-P\|_{0} \leq C h^{k}\left(\mu|\boldsymbol{u}|_{k+1}+|p|_{k}\right)+\frac{C}{\mu} \llbracket \boldsymbol{u}-\boldsymbol{U} \rrbracket,
\end{gathered}
$$

where $(\boldsymbol{u}, p)$ is the solution of (2.4), (2.2), (2.3) and the constant $C$ depends upon $f$, but not on $h$ or $\mu$.

Proof. It follows from (6.20), 6.19) and (6.15) that the factor of $\mu$ in the left-hand side of (6.16) is greater than or equal to

$$
K \mu \llbracket \chi \chi \rrbracket^{2}\left(1-\frac{N(h) C(4)}{K^{2} \mu^{2}}\right)\|\boldsymbol{f}\|_{L^{4 / 3}(\Omega)} \geq \frac{1}{2} K \mu \llbracket \chi \chi \rrbracket^{2} .
$$

Then substituting this lower bound into (6.16) and dividing both sides by $\frac{1}{2} K \mu$, we obtain

$$
\begin{aligned}
\| \boldsymbol{\chi} \rrbracket^{2}+\frac{1}{K \mu}\left(\sum_{E \in \mathcal{E}_{h}} \int_{\partial E_{-}}\left|\left\{\boldsymbol{U}_{I}\right\} \cdot \boldsymbol{n}_{E}\right|\left\|\boldsymbol{\chi}^{\mathrm{ext}}-\boldsymbol{\chi}^{\mathrm{int}}\right\|^{2}+\int_{\Gamma_{+}}|\boldsymbol{U} \cdot \boldsymbol{n}|\|\boldsymbol{\chi}\|^{2}\right) \\
\leq \frac{2}{K}\left(a\left(\boldsymbol{u}-\boldsymbol{U}_{I}, \boldsymbol{\chi}\right)+J_{0}\left(\boldsymbol{u}-\boldsymbol{U}_{I}, \boldsymbol{\chi}\right)+\frac{1}{\mu} b\left(\boldsymbol{\chi}, P_{I}-p\right)\right) \\
+\frac{2}{K \mu}\left(c\left(\boldsymbol{u}-\boldsymbol{U}_{I} ; \boldsymbol{U}_{I}, \boldsymbol{\chi}\right)+c\left(\boldsymbol{u} ; \boldsymbol{U}_{I}-\boldsymbol{u}, \boldsymbol{\chi}\right)\right) .
\end{aligned}
$$

The first group of terms in the right-hand side is estimated in Theorem 5.1 and the second group of terms is estimated in Remark 6.5. Then the theorem follows from these two estimates, the approximating properties of $\boldsymbol{P}_{h}$ and the triangle inequality. The estimate for the pressure follows from the inf-sup condition.

Of course, if 6.21) holds, then the solution $\boldsymbol{u}$ of the exact problem is unique. As expected, the $L^{2}$ error estimate for the symmetric formulation is optimal.

Theorem 6.7. Under the assumptions of Theorem 6.6, and if $\Omega$ is convex, there is a constant $C$ independent of $h$ such that

$$
\|\boldsymbol{u}-\boldsymbol{U}\|_{0, \Omega} \leq C h^{k+1} .
$$

Proof. For the sake of brevity, let us sketch the proof. Its main idea, due to [26], consists of two steps. First, we solve the auxiliary discrete Stokes problem

$$
\begin{gathered}
\mu\left(a\left(\boldsymbol{w}_{h}, \boldsymbol{v}_{h}\right)+J_{0}\left(\boldsymbol{w}_{h}, \boldsymbol{v}_{h}\right)\right)+b\left(\boldsymbol{v}_{h}, z_{h}\right)=\left(\boldsymbol{f}, \boldsymbol{v}_{h}\right)-c\left(\boldsymbol{u} ; \boldsymbol{u}, \boldsymbol{v}_{h}\right), \quad \forall \boldsymbol{v}_{h} \in \boldsymbol{X}_{h}, \\
b\left(\boldsymbol{w}_{h}, q_{h}\right)=0, \quad \forall q_{h} \in M_{h},
\end{gathered}
$$

and we observe that the exact solution is precisely $(\boldsymbol{u}, p)$. As in Theorem [5.3, the error $\boldsymbol{w}_{h}-\boldsymbol{u}$ in the energy and $L^{2}$ norms is optimal. The second step consists of estimating $\left\|\boldsymbol{U}-\boldsymbol{w}_{h}\right\|_{0, \Omega}$. If $\boldsymbol{\xi}=\boldsymbol{U}-\boldsymbol{w}_{h}$, the error equation can be rewritten as (6.24)

$$
\mu\left(a(\boldsymbol{\xi}, \boldsymbol{\xi})+J_{0}(\boldsymbol{\xi}, \boldsymbol{\xi})\right)+c(\boldsymbol{u} ; \boldsymbol{\xi}, \boldsymbol{\xi})+c(\boldsymbol{\xi} ; \boldsymbol{U}, \boldsymbol{\xi})=c\left(\boldsymbol{u} ; \boldsymbol{u}-\boldsymbol{w}_{h}, \boldsymbol{\xi}\right)+c\left(\boldsymbol{u}-\boldsymbol{w}_{h} ; \boldsymbol{U}, \boldsymbol{\xi}\right) .
$$


The left-hand side of (6.24) is similar to that of (6.16) and has the same lower bound. Then, we prove that its right-hand side is bounded by $C h^{k+1} \llbracket \boldsymbol{\xi} \rrbracket$.

\section{Conclusion}

In this paper, we have established optimal a priori estimates for a totally discontinuous family of approximations of the steady incompressible Stokes and NavierStokes equations in two dimensions. We have balanced the discontinuities by inserting suitable jump terms and in the nonsymmetric case these have only the viscosity as a coefficient. Simultaneously, we treat domain decomposition with nonmatching grids. The schemes are locally conservative away from subdomain interfaces. To our knowledge, this is the first analysis of discontinuous Galerkin methods with

nonmatching domain decomposition for Stokes and Navier-Stokes equations in the primitive variables.

\section{REFERENCES}

1. R. A. Adams, Sobolev Spaces, Academic Press, New York, NY (1975). MR 56:9247

2. G. A. Baker, W. N. Jureidini and O. A. Karakashian, Piecewise solenoidal vector fields and the Stokes problem, SIAM J. Numer. Anal. 27 (1987), pp. 1466-1485. MR 91m:65246

3. R. Becker, P. Hansbo and R. Stenberg, A finite element method for domain decomposition with nonmatching grids, M2AN 37 (2003), pp. 209-225.

4. P. Ciarlet, The finite element methods for elliptic problems, North-Holland, Amsterdam (1978). MR 58:25001

5. B. Cockburn, G. Kanschat, D. Schotzau and C. Schwab, Local discontinuous Galerkin methods for the Stokes system, SIAM J. Numer. Anal. 40 (2002) pp. 319-343. MR 2003g:65141

6. M. Crouzeix and R. S. Falk, Nonconforming finite elements for the Stokes problem, Math. Comp. 52 (186) (1989), pp. 437-456. MR 89i:65113

7. M. Crouzeix and P. A. Raviart, Conforming and nonconforming finite element methods for solving the stationary Stokes equations, R.A.I.R.O. Numerical Analysis R3 (1973), pp. 33-76. MR 49:8401

8. M. Fortin, An analysis of the convergence of mixed finite element methods, R.A.I.R.O. Numerical Analysis 11 (1977), pp. 341-354. MR 57:4473

9. M. Fortin and M. Soulié, A nonconforming piecewise quadratic finite element on triangles, International Journal for Numerical Methods in Engineering 19 (1983), pp. 505-520. MR 84g:76004

10. V. Girault and J.-L. Lions, Two-grid finite-element schemes for the steady Navier-Stokes problem in polyhedra, Port. Math. (N.S.) 58 (2001), no. 1, pp. 25-57. MR 2002m:65117

11. V. Girault, R. Glowinski, H. López and J.-P. Vila, A boundary multiplier/fictitious domain method for the steady incompressible Navier-Stokes equations, Numer. Math. 88 (2001), no. 1, pp. 75-103. MR 2002b:65166

12. V. Girault and P. A. Raviart, Finite element methods for Navier-Stokes equations: theory and algorithms, Springer Series in Computational Mathematics 5 (1986). MR 88b:65129

13. V. Girault and R. L. Scott, A quasi-local interpolation operator preserving the discrete divergence, Calcolo 40 (2003), pp. 1-19.

14. P. Grisvard, Elliptic problems in nonsmooth domains, Pitman Monographs and Studies in Mathematics 24, Pitman, Boston, MA (1985). MR 86m:35044

15. P. Houston, C. Schwab and E. Suli, Discontinuous hp-finite element methods for advectiondiffusion problems, SIAM J. Numer. Anal. 39 (2002), no. 6, pp. 2133-2163. MR 2003d:65108

16. O. A. Karakashian and W. Jureidini, A nonconforming finite element method for the stationary Navier-Stokes equations, SIAM J. Numer. Anal. 35 (1998), pp. 93-120. MR 99d:65320

17. P. Lesaint and P. A. Raviart, On a finite element method for solving the neutron transport equation, In: Mathematical Aspects of Finite Element Methods in Partial Differential Equations, C. A. de Boor (Ed.), Academic Press, (1974) pp. 89-123. MR 58:31918

18. J. L. Lions and E. Magenes, Problèmes aux Limites non Homogènes et Applications, I, Dunod, Paris (1968). MR 40:512 
19. J. L. Lions, Quelques méthodes de résolution des problèmes aux limites non linéaires, Dunod, Paris (1969). MR 41:4326

20. J. T. Oden, I. Babuška, and C. E. Baumann, A discontinous hp finite element method for diffusion problems, Journal of Computational Physics 146 (1998) pp. 491-519. MR 99m:65173

21. O. Pironneau, Finite Elements for Fluids, Wiley, Chichester (1989). MR 90j:76016

22. B. Rivière, M. F. Wheeler and V. Girault, Improved energy estimates for interior penalty, constrained and discontinuous Galerkin methods for elliptic problems. Part I, Computational Geosciences 3 (1999) pp. 337-360. MR 2001d:65145

23. B. Rivière, M. F. Wheeler and V. Girault, A priori error estimates for finite element methods based on discontinuous approximation spaces for elliptic problems, SIAM J. Numer. Anal., 39 (3) (2001) pp. 902-931. MR 2002g:65149

24. B. Rivière and M. F. Wheeler, Nonconforming methods for transport with nonlinear reaction, Proceedings of the Joint Summer Research Conference on Fluid Flow and Transport in Porous Media (2001), Contemp. Math., vol. 295, Amer. Math. Soc., Providence, RI, 2002, pp. 421-432.

25. R. Temam, Navier-Stokes equations. Theory and numerical analysis. Reprint of the 1984 edition. AMS Chelsea Publishing, Providence, RI, 2001. MR 2002j:76001

26. M. F. Wheeler, A priori $L^{2}$ error estimates for Galerkin approximations to parabolic partial differential equations, SIAM J. Numer. Anal. 10 (4) (1973) pp. 723-759. MR 50:3613

27. M. F. Wheeler, An elliptic collocation-finite element method with interior penalties, SIAM J. Numer. Anal. 15 (1) (1978) pp. 152-161. MR 57:11117

Université Pierre et Marie Curie, Paris Vi, Laboratoire Jacques-Louis Lions, 4, Place Jussieu, F-75230 Paris Cedex 05, France

E-mail address: girault@ann.jussieu.fr

Department of Mathematics, University of Pittsburgh, 301 Thackeray, Pittsburgh, Pennsylvania 15260

E-mail address: riviere@math.pitt.edu

The Center for Subsurface Modeling, Institute for Computational Engineering and Sciences, The University of Texas, 201 E. 24th St., Austin, Texas 78712

E-mail address: $\mathrm{mf}$ w@ices.utexas.edu 